\title{
Completed representation ring spectra of nilpotent groups
}

\author{
TYLER LAWSON
}

In this paper, we examine the "derived completion" of the representation ring of a pro- $p$ group $\mathcal{G}_{p}^{\wedge}$ with respect to an augmentation ideal. This completion is no longer a ring: it is a spectrum with the structure of a module spectrum over the Eilenberg-MacLane spectrum $\mathbb{H} \mathbb{Z}$, and can have higher homotopy information. In order to explain the origin of some of these higher homotopy classes, we define a deformation representation ring functor $R[-]$ from groups to ring spectra, and show that the map $R\left[\mathcal{G}_{p}^{\wedge}\right] \rightarrow R[\mathcal{G}]$ becomes an equivalence after completion when $\mathcal{G}$ is finitely generated nilpotent. As an application, we compute the derived completion of the representation ring of the simplest nontrivial case, the $p$-adic Heisenberg group.

55P60; 55P43, 19A22

\section{Introduction}

The original Adams spectral sequence provided a streamlined method for computing the $p$-primary part of the stable homotopy groups of spheres, or more generally for attempting to compute the group of maps between two objects in the stable homotopy category. This process has seen highly useful generalizations, such as the AdamsNovikov spectral sequence exposited in Adams [1], and the $E$-nilpotent completion spectral sequence of Bousfield [3]. By using the same method of proof, Carlsson gave a construction for modules over a ring $R$ in [5]. The underlying method is that given an object $X$ and a generalized homology theory $E$, one constructs a "derived completion", or $E$-nilpotent completion, which is a pro-object $X_{E}^{\wedge}$, and there is a spectral sequence for computing the space of maps from an object $Y$ into $X_{E}^{\wedge}$. In the case where $X$ is a finitely generated module over a Noetherian ring and $E$ is the homology theory $\operatorname{Tor}_{*}^{R}(R / I,-)$, the completion $X_{E}^{\wedge}$ is equivalent to the $I$-adic completion of $X$.

With the advent of highly structured categories of modules over algebra spectra, such as those constructed by Elmendorf-Kriz-Mandell-May [7], it has become possible to construct an Adams spectral sequence generalizing all of the above constructions, as in Baker-Lazarev [2]. For example, by [7, Theorem IV.2.4], the derived category of modules over a ring $R$ is equivalent to the homotopy category of module spectra 
over the Eilenberg-MacLane spectrum $\mathbb{H} R$. (Throughout this paper, we will use the

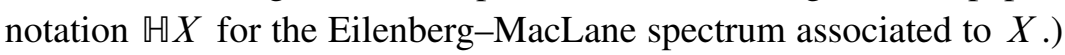

In this paper, we will be mainly focused on the case where $M=R$, as an analogue of completing a ring with respect to an ideal with quotient ring $E$. When $E$ is an $R$-algebra, the $E$-nilpotent completion of $R$ (as an $R$-module) can be canonically expressed as the totalization of the cobar complex

$$
\left\{E \underset{R}{\wedge} \stackrel{\wedge}{R}^{\prime} E\right\}
$$

with coface and codegeneracy maps given by the unit map $R \rightarrow E$ and multiplication $E \wedge_{R} E \rightarrow E$ respectively. (A different characterization, and another canonical construction, will be given in Section 2.) We write $R_{E}^{\wedge}$ for this "derived completion" of $R$ as an $R$-module spectrum.

Our interest is in examining this completion functor in some specific cases of modules over a non-Noetherian ring. Let $R$ be a ring, and $E$ and $M$ chain complexes of left $R$-modules. Then the completion $M_{E}^{\wedge}$ is a pro-object in the derived category of $R$-modules, or equivalently a pro-weak equivalence class of chain complexes. Under certain algebraic conditions, including $\operatorname{Tor}_{*}^{R}(E, E)$ being flat over $H_{*} E$, there is a spectral sequence

$$
\operatorname{Ext}_{\mathrm{Ext}_{R}^{* *}(E, E)}^{* *}\left(\operatorname{Ext}_{R}^{*}(M, E), H^{*}(E)\right)
$$

that abuts to the homology groups of $M_{E}^{\wedge}$. Convergence is a more delicate question; for example, see Baker-Lazarev [2].

One of the first interesting examples has to do with the relationship between circle actions and actions of finite cyclic groups. Specifically, let $\mathbb{Z}_{p}$ be the group of $p$-adic integers under addition, and let $R\left[\mathbb{Z}_{p}\right]$ be its representation ring. This is the free abelian group on irreducible continuous complex representations of $\mathbb{Z}_{p}$, and as such is isomorphic to the group ring $\mathbb{Z}\left[\mu_{p^{\infty}}\right]$, the group ring on the group $\mu_{p^{\infty}}$ of $p$-power roots of unity in $\mathbb{C}^{\times}$. If $E$ is the $R\left[\mathbb{Z}_{p}\right]$-module $\mathbb{Z} / p$ with trivial action, one can compute with the above spectral sequence and find that

$$
H_{*}\left(R\left[\mathbb{Z}_{p}\right]_{\mathbb{Z} / p}^{\wedge}\right) \cong \begin{cases}\mathbb{Z}_{p} & \text { if } *=0,1 \\ 0 & \text { otherwise. }\end{cases}
$$

The computation of the completion of the representation ring of the $p$-adic Heisenberg group $H_{p}^{\wedge}$ with respect to the $R\left[H_{p}^{\wedge}\right]$-module $\mathbb{Z} / p$ was initiated by $\mathrm{C}$ Bray in [4], 
and completed here. The result takes the following form:

$$
H_{*}\left(R\left[H_{p}^{\wedge}\right]_{\mathbb{Z} / p}^{\wedge}\right) \cong \begin{cases}\widehat{\oplus}_{\zeta \in \mu_{p} \infty} \mathbb{Z}_{p} & \text { if } *=0 \\ \widehat{\oplus}_{\zeta \in \mu_{p} \infty} \mathbb{Z}_{p} \oplus \mathbb{Z}_{p} & \text { if } *=1 \\ \widehat{\oplus}_{\zeta \in \mu_{p} \infty} \mathbb{Z}_{p} & \text { if } *=2 \\ 0 & \text { otherwise. }\end{cases}
$$

(Here $\widehat{\oplus}$ denotes the completed direct sum of $p$-complete modules.) This computation can be carried out using the spectral sequence of equation (1). As such, it involves a significant number of extension problems, especially with respect to determining an algebra structure possessed by $R\left[H_{p}^{\wedge}\right]_{\mathbb{Z} / p}^{\wedge}$

The purpose of this paper is to explore the origin of higher homology classes in the case of $R\left[\mathcal{G}_{p}^{\wedge}\right.$, where $\mathcal{G}$ is a finitely generated nilpotent group and $\mathcal{G}_{p}^{\wedge}$ is its pro- $p$ completion. Our contention is that some of this higher homotopy arises from geometric information about the representation theory of $\mathcal{G}$.

Specifically, let $\operatorname{Irr}(\mathcal{G})$ be the set of isomorphism classes of irreducible representations of $\mathcal{G} . \operatorname{Irr}(\mathcal{G})$ comes naturally equipped with the structure of a topological space, and there is a natural map $\operatorname{Irr}\left(\mathcal{G}_{p}^{\wedge}\right) \rightarrow \operatorname{Irr}(\mathcal{G})$, where $\operatorname{Irr}\left(\mathcal{G}_{p}^{\wedge}\right)$ has the discrete topology (Both of these topologies arise naturally from the starting point of the compact-open topology on mapping spaces.) Applying $\mathbb{R} \mathbb{Z} \wedge \Sigma_{+}^{\infty}(-)$, where $\Sigma_{+}^{\infty}$ denotes the unbased suspension spectrum functor, gives a map

$$
\mathbb{H} R\left[\mathcal{G}_{p}^{\wedge}\right] \rightarrow \mathbb{Z} \wedge \Sigma_{+}^{\infty} \operatorname{Irr}(\mathcal{G})
$$

of $\mathbb{H Z Z Z - m o d u l e s . ~}$

Proposition 1 If $\mathcal{G}$ is a finitely generated, nilpotent, discrete group, there exists a natural map of $\mathbb{S}$-algebras realizing the map of equation (4).

Proof In Section 4, we construct a map of commutative monoids in symmetric spectra $R\left[\mathcal{G}_{p}^{\wedge}\right] \rightarrow R[\mathcal{G}]$, which can be realized as a map of $\mathbb{S}$-algebras. Section 5, Proposition 38 and Corollary 39 show this is equivalent to the map of equation (4) in the case where $\mathcal{G}$ is finitely generated, nilpotent, and discrete.

Our main result is the following.

Theorem 2 The map $\mathbb{R}\left[\mathcal{G}_{p}^{\wedge}\right] \rightarrow \mathbb{Z} \wedge \Sigma_{+}^{\infty}(\operatorname{Irr}(\mathcal{G}))$ induces an equivalence of derived completions

$$
\mathbb{H} R\left[\mathcal{G}_{p}^{\wedge}\right]_{\mathfrak{M} \mathbb{F}_{p}}^{\wedge} \rightarrow \mathbb{Z} \wedge \Sigma_{+}^{\infty}(\operatorname{Irr}(\mathcal{G}))_{\mathbb{H} \mathbb{F}_{p}}^{\wedge},
$$


where the derived completion on the left is taken as modules over $\mathbb{H} R\left[\mathcal{G}_{p}^{\wedge}\right]$ and on the right as modules over $\mathbb{Z} \mathbb{Z} \wedge \Sigma_{+}^{\infty}(\operatorname{Irr}(\mathcal{G}))$.

The proof requires some intermediate results. We first make the following definition.

Definition 3 Suppose $E$ is a fixed commutative connective $\mathbb{S}$-algebra. A map $A \rightarrow A^{\prime}$ of commutative $\mathbb{S}$-algebras over $E$ is a multiplicative $E$-equivalence if the map $E \rightarrow A^{\prime} \wedge_{A} E$ is a equivalence.

In Section 2 we will recall some definitions and properties of Bousfield localization. A multiplicative $E$-equivalence $A \rightarrow A^{\prime}$ is also an ordinary $E$-equivalence of $A-$ modules, and hence $A$ and $A^{\prime}$ have the same $E$-localizations as $A$-modules. More is true, however; by Proposition 12 , the $E$-localization of $A$ as an $A$-module is homotopy equivalent to the $E$-localization of $A^{\prime}$ as an $A^{\prime}$-module.

The $E$-localization $L_{E}^{A}(A)$ of $A$ and the derived completion $A_{E}^{\wedge}$ are related by a map $L_{E}^{A}(A) \rightarrow A_{E}^{\wedge}$, where the localization is viewed as a constant pro-object. The $E$-localization of $A$ is equivalent to the homotopy $\operatorname{limit} \operatorname{holim}\left(A_{E}^{\wedge}\right)$ under $\operatorname{good}$ circumstances, such as when smashing with $E$ can be passed under the limit.

Theorem 4 Suppose $\mathcal{G}$ is a finitely generated discrete group. The map $\mathbb{R} R\left[\mathcal{G}_{p}^{\wedge}\right] \rightarrow$ $\mathbb{U} \mathbb{Z} \wedge \Sigma_{+}^{\infty}(\operatorname{Irr}(\mathcal{G}))$ is a multiplicative $\mathbb{M F}_{p}$-equivalence.

Proof By Proposition 38 and Corollary 39, this statement is equivalent to Proposition 40 .

The results leading to Theorem 4 are proved by making use of the structure of these $\mathbb{S}$-algebras as algebra spectra over $R\left[\mathcal{G}_{\mathrm{ab}}^{\wedge}\right]$, the representation ring of the abelianization of the profinite completion of $\mathcal{G}$.

Theorem 2 then follows from Theorem 4 and Lemma 18, which proves that multiplicative $E$-equivalences induce equivalences of derived completions. In fact, the groups listed in equation (3) will be proven in Section 6 to be the homotopy groups of the $\mathbb{M F}$-localization of $R\left[H_{p}^{\wedge}\right]$.

We now briefly outline the stages of the argument. In Section 2 we will show that multiplicative $E$-equivalences are closed under composition in the category of $\mathbb{S}$ algebras over $E$. Additionally, we show in Proposition 12 that if $R$ is a commutative S-algebra, and $A \rightarrow A^{\prime}$ is a map of commutative $R$-algebras over $E$ which is an $E$-equivalence as $R$-modules, then this map is also a multiplicative $E$-equivalence. 
In Section 3 we recall and apply results of Lubotzky-Magid [9] about the spaces of representations associated to nilpotent groups. Section 4 models the map of equation (4) by a naturally defined map of ring objects in $\Gamma$-spaces, obtained by considering spaces of representations. The proof of Theorem 2 is the main content of Section 5.

The $\mathbb{S}$-algebra $\mathbb{Z} \mathbb{Z} \wedge \Sigma_{+}^{\infty}(\operatorname{Irr}(\mathcal{G}))$ is more tractable. In Section 6, this spectrum is used to compute the homotopy groups of the Bousfield localization of the representation ring of $H_{p}^{\wedge}$, the $p$-adic Heisenberg group. The results agree with the computation referenced in equation (3), with the additional benefit that the algebra structure becomes apparent. The completion of this computation appears in Section 6.3: the homology of the Bousfield localization of $R\left[H_{p}^{\wedge}\right]$ is a retract of the $p$-completion of $H_{*}(\operatorname{Irr}(H))$, the homology of the space of irreducible representations of the integral Heisenberg group. The retract contains precisely those summands of the homology corresponding to components that contain pro- $p$ representations. Finally, in Section 6.4, these groups are shown to coincide with the homology groups of the derived completion $R\left[H_{p}^{\wedge}\right]_{\mathfrak{U} \mathbb{F}_{p}}^{\wedge}$.

Acknowledgements The author would like to thank Daniel Bump, Veronique Godin, Robert Guralnick, Haynes Miller, and Daniel Ramras for helpful discussions related to this paper. The majority of this research was carried out while the author was a student of Gunnar Carlsson, and the present work owes much to him. The author was partially supported by NSF award 0402950 and the ARCS foundation.

\section{Localizations of module spectra}

Throughout this section, we will be working in the category $\mathcal{M}_{R}$ of module spectra over a fixed commutative $\mathbb{S}$-algebra $R$, and $E$ will denote an element of $\mathcal{M}_{R}$. (In the applications, $E$ will be a "quotient" $R$-algebra with unit $\eta: R \rightarrow E$.) See ElmendorfKriz-Mandell-May [7] for foundational material on these categories of spectra. Our real interest lies in the homotopy category $\mathcal{D}_{R}$, which is closed symmetric monoidal under $\wedge_{R}$ and $F_{R}(-,-)$, and so we will usually replace $R$-modules by cell $R-$ modules without comment. The book Hovey-Palmieri-Strickland [8] serves as a good introduction to the abstract theory of these homotopy categories.

We will write $M_{*}, M_{*}(N)$, and $[M, N]^{*}$ for the groups $\pi_{*}(M), \pi_{*}\left(M \wedge_{R} N\right)$, and $\pi_{-*}\left(F_{R}(N, M)\right)$ respectively, where $F_{R}(N, M)$ is the $R$-module function spectrum. (The underlying $\mathbb{S}$-algebra $R$ will be specified if it is ambiguous.)

In the setting of ring spectra, one appropriate replacement for the notion of "completion at an ideal" is Bousfield localization. The $E$-localization of an $R$-module $M$ is 
obtained from $M$ by throwing away all information that is not determined by the homology theory $E_{*}(-)$.

We begin by recalling some definitions.

Definition 5 An $R$-module $X$ is $E$-acyclic if $E \wedge_{R} X \simeq *$.

Definition 6 A map $f: M \rightarrow N$ of $R$-modules is an $E$-equivalence if the cofiber is $E$-acyclic.

Definition 7 An object $M$ is $E$-local if $[X, M]_{R}^{*}=0$ whenever $X$ is $E$-acyclic. ( $E$-acyclic objects are closed under suspension, so this is equivalent to requiring that $[X, M]_{R}=0$ for all $E$-acyclic $X$.)

Remark 8 The definition immediately implies that $E$-local objects form a thick subcategory of $\mathcal{D}_{R}$ closed under homotopy limits. If $E$ is an $R$-algebra, then the adjunction $\left[E \wedge_{R} X, M\right]_{E} \cong[X, M]_{R}$ implies that all $E$-module spectra $M$ are E-local.

Definition 9 An $E$-localization of $M$ is an $E$-local object $L_{E}(M)$ equipped with an $E$-equivalence $M \rightarrow L_{E}(M)$. If the underlying $\mathbb{S}$-algebra $R$ is ambiguous, we write $L_{E}^{R}(M)$.

The definition implies that any map from $M$ to a local object $N$ factors uniquely (up to homotopy) through $L_{E}(M)$, and hence any such localization is unique up to equivalence. Therefore, an $E$-equivalence $M \rightarrow M^{\prime}$ gives rise to an equivalence $L_{E}(M) \rightarrow L_{E}\left(M^{\prime}\right)$.

For details about the existence of localizations, consult [7, Chapter VIII]. A model structure exists on the category of $R$-modules that has $E$-equivalences as weak equivalences, and the fibrant objects in this model structure become $E$-local in the homotopy category of $R$-modules. For our purposes, however, only existence is important.

We will now list some consequences about maps of $\mathbb{S}$-algebras and their behavior under localization.

Lemma 10 Suppose $R \rightarrow R^{\prime}$ is a map of commutative $S$-algebras, and $E$ is an $R-$ module. Define $F=E \wedge_{R} R^{\prime}$. Then for any $R^{\prime}$-module $M$, the map $M \rightarrow L_{F}^{R^{\prime}}(M)$ is an $E$-localization of $M$ as an $R$-module.

Proof This is [7, Proposition VIII.1.8]. 
Lemma 11 Suppose $R \rightarrow R^{\prime}$ is a map of commutative $\mathbb{S}$-algebras and $E^{\prime}$ is an $R^{\prime}$-module. If $M$ is an $R^{\prime}$-module that is $E^{\prime}$-acyclic as an $R$-module, then $M$ is $E^{\prime}$-acyclic as an $R^{\prime}$-module.

Proof Without loss of generality, assume $R$ is a $q$-cofibrant commutative $\mathbb{S}$-algebra, $R^{\prime}$ is a commutative $q$-cofibrant $R$-algebra, and $E^{\prime}$ is a cell $R^{\prime}$-module. By [7, Proposition IX.2.3], $E^{\prime} \wedge R^{\prime} M$ is equivalent to $B^{R}\left(E^{\prime}, R^{\prime}, M\right)$, the geometric realization of the simplicial spectrum

$$
B_{p}^{R}\left(E^{\prime}, R^{\prime}, M\right)=E^{\prime}{\underset{R}{\wedge}}_{\left(R^{\prime}\right)^{\wedge}{ }_{R}(p)}{ }_{R}^{\wedge} M .
$$

The symmetric monoidal properties of the smash product in $\mathcal{M}_{R}$ imply that $B_{p}^{R}\left(E^{\prime}, R^{\prime}, M\right) \simeq *$ because $E^{\prime} \wedge_{R} M \simeq *$. Therefore, $E^{\prime} \wedge_{R^{\prime}} M \simeq *$.

Proposition 12 Suppose there is a diagram $R \rightarrow A \rightarrow A^{\prime}$ of maps of commutative $S$-algebras, and $E$ is an $A^{\prime}$-module. There is a natural (up to homotopy) map $L_{E}^{A}(A) \rightarrow L_{E}^{A^{\prime}}\left(A^{\prime}\right)$. If $A \rightarrow A^{\prime}$ is an $E$-equivalence as $R$-modules, then this map is an equivalence.

Proof The object $L_{E}^{A^{\prime}}\left(A^{\prime}\right)$ is $E$-local as an $A$-module; if $X$ is an $E$-acylic $A-$ module, $A^{\prime} \wedge_{A} X$ is an $E$-acyclic $A^{\prime}$-module, and hence

$$
\left[X, L_{E}^{A^{\prime}}\left(A^{\prime}\right)\right]_{A} \cong\left[A^{\prime} \underset{A}{\wedge} X, L_{E}^{A^{\prime}}\left(A^{\prime}\right)\right]_{A^{\prime}}=0
$$

Therefore, the composite map $A \rightarrow A^{\prime} \rightarrow L_{E}^{A^{\prime}}\left(A^{\prime}\right)$ factors through $L_{E}^{A}(A)$.

Suppose the map $A \rightarrow A^{\prime}$ is an $E$-equivalence of $R$-modules, or equivalently the cofiber is $E$-acyclic as an $R$-module. By Lemma 11 , the cofiber is $E$-acyclic as an $A$-module, so without loss of generality we can assume $R=A$.

Define $F=E \wedge_{A} A^{\prime}$. Because the map $A \rightarrow A^{\prime}$ is an $E$-equivalence, the maps

$$
E \underset{A}{\wedge} A \rightarrow E \underset{A}{\wedge} A^{\prime} \rightarrow E
$$

are all equivalences, where the right-hand map is the multiplication map for $A^{\prime}$-modules. This is an $A^{\prime}$-module map, so $F \simeq E$ as an $A^{\prime}$-module. Thus

$$
L_{E}^{A}(A) \simeq L_{E}^{A}\left(A^{\prime}\right) \simeq L_{F}^{A^{\prime}}\left(A^{\prime}\right) \simeq L_{E}^{A^{\prime}}\left(A^{\prime}\right),
$$

by Lemma 10 .

Lemma 13 The collection of commutative $\mathbb{S}$-algebras over $E$, with multiplicative $E$-equivalences as maps, forms a category. 
Proof Suppose $A^{\prime \prime} \rightarrow A \rightarrow A^{\prime}$ are both multiplicative $E$-equivalences. Consider the following diagram of maps.

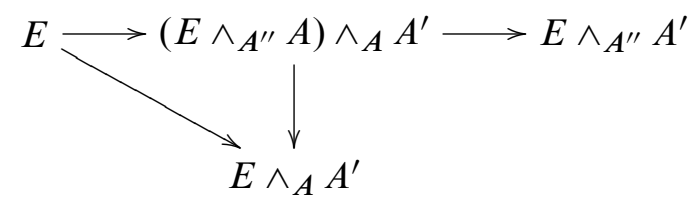

The lower maps are equivalences by assumption, while the rightmost map is an isomorphism by associativity of the smash product.

Let $R$ be a commutative $\mathbb{S}$-algebra and $E$ an $R$-algebra. In [3], the $E$-nilpotent completion (or derived completion) of an ordinary spectrum was defined; we will briefly recall this definition in the context of modules over $R$.

Definition 14 The family of $E$-nilpotent objects is the thick subcategory of the category of $R$-modules generated by $E$-modules, i.e. the smallest full subcategory containing the $E$-modules closed under retracts and exact triangles.

Definition 15 An $E$-nilpotent completion of an $R$-module $M$ is an inverse system $\left\{W_{s}\right\}_{s \geq 0}$ of $E$-nilpotent objects under $M$ such that the map

$$
\lim \left[W_{s}, X\right]_{R} \rightarrow[M, X]_{R}
$$

is an isomorphism for any $E$-nilpotent object $X$. (The $E$-nilpotent completion of $M$ is defined up to pro-equivalence.)

Remark 16 Note that in order to check that an object is an $E$-nilpotent completion of $M$, it suffices to check the isomorphism $\lim \left[W_{S}, X\right]_{R} \rightarrow[M, X]_{R}$ when $X$ is an $E$-module, as both sides preserve retracts and exact triangles.

Remark $17 E$-nilpotent completions exist; they can be explicitly constructed by the following standard procedure. Let $I$ be the fiber of the map $R \rightarrow E$, and $I^{(s)}$ its $s$-fold smash power over $R$. Let $R / I^{(s)}$ be the cofiber of the map $I^{(s)} \rightarrow R^{(s)} \cong R$. One finds that there are cofiber sequences

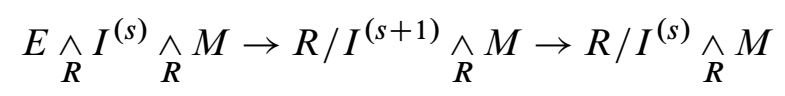

for all $s \geq 0$. By induction, we find that the $R / I^{(s)} \wedge_{R} M$ are $E$-nilpotent. In addition, if $X$ is an $E$-module, the adjunction

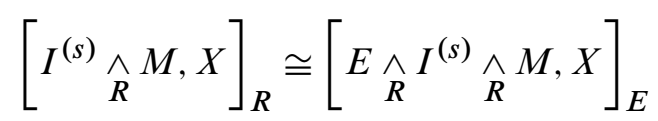


shows that any map in $\left[R / I^{(s)} \wedge_{R} M, X\right]_{R}$ that goes to zero in $[R, M]_{R}$ factors through $\left[\Sigma E \wedge_{R} I^{(s)} \wedge_{R} M, X\right]_{R}$, which goes to zero in $\left[R / I^{(s+1)} \wedge_{R} M, X\right]_{R}$. Additionally, the map $[R / I, X]_{R} \rightarrow[R, X]_{R}$ is already surjective by the same adjunction.

Lemma 18 If $M \rightarrow N$ is an $E$-equivalence of $R$-modules, then the map of derived completions $M_{E}^{\wedge} \simeq N_{E}^{\wedge}$ is an equivalence of pro-objects.

Proof Let $\left\{W_{s}\right\}$ be an $E$-nilpotent completion of $N$. If $X$ is an $E$-module, then $X$ is $E$-local, so the composite map

$$
\lim \left[W_{s}, X\right]_{R} \rightarrow[N, X]_{R} \rightarrow[M, X]_{R}
$$

is an isomorphism.

\section{Representations of nilpotent groups}

Let $\mathcal{G}$ be a topological group, and let $\operatorname{Hom}(\mathcal{G}, \mathrm{U}(n))$ be the space of (continuous) group homomorphisms from $\mathcal{G}$ to $\mathrm{U}(n)$, considered as a subspace of the mapping space $\operatorname{Map}(\mathcal{G}, \mathrm{U}(n))$. The latter space is given the compact-open topology.

Example 19 (Finitely generated discrete groups) Suppose $\mathcal{G}$ is a finitely generated discrete group, with presentation

$$
\mathcal{G}=\left\langle g_{1}, g_{2}, \ldots \mid r_{1}\left(g_{i}\right)=r_{2}\left(g_{i}\right)=\ldots=1\right\rangle .
$$

Then $\operatorname{Hom}(\mathcal{G}, \mathrm{U}(n))$ is the set of real points of an algebraic variety, as follows. Writing a general complex matrix in the form $A=\left(a_{j k}+b_{j k} i\right)_{j k}$, we can expand out the formula $A A^{*}=I$. We find that the points of $\mathrm{U}(n)$ are the real points of the algebraic variety

$$
\left\{\left(a_{j k}, b_{j k}\right) \mid \sum_{\ell} a_{j \ell} a_{k \ell}+b_{j \ell} b_{k \ell}=\delta_{j k}, \sum_{\ell} b_{j \ell} a_{k \ell}-a_{j \ell} b_{k \ell}=0\right\} .
$$

The space $\operatorname{Hom}(\mathcal{G}, \mathrm{U}(n))$ can be written as the set

$$
\left\{\left(A_{1}, A_{2}, \ldots\right) \in \prod \mathrm{U}(n) \mid r_{1}\left(A_{i}\right)=r_{2}\left(A_{i}\right)=\ldots=I\right\},
$$

and the mapping topology coincides with the subspace topology from $\prod \mathrm{U}(n)$. (Pointwise convergence of homomorphisms is the same as pointwise convergence on the generating set.) The conditions $r_{j}\left(A_{i}\right)=I$ introduce extra equations that the points 
must satisfy, but these are still algebraic. Therefore, the space $\operatorname{Hom}(\mathcal{G}, \mathrm{U}(n))$ is a closed subvariety of $\prod \mathrm{U}(n)$.

Example 20 (Profinite groups) First, we will prove that there exists a sufficiently small open neighborhood $\mathcal{U}$ of the identity in $\mathrm{U}(n)$ that contains no nontrivial subgroups. Let $\mathcal{V}$ be a ball centered at the identity of the Lie algebra $\mathfrak{u}(n)$ such that exp: $\mathfrak{u}(n) \rightarrow$ $\mathrm{U}(n)$ is injective on $2 \mathcal{V}$, and let $\mathcal{U}=\exp (\mathcal{V})$. For any $I \neq g \in \mathcal{U}, g=\exp (A)$ for some $A \in \mathcal{V} . A \neq 0$, so there exists a unique nonnegative integer $n$ such that $2^{n-1} A \in \mathcal{V}$ but $2^{n} A \notin \mathcal{V}$. Then, because $2^{n} A \in 2 \mathcal{V}$ where the exponential map is injective, we find that $g^{2^{n}}=\exp \left(2^{n} A\right)$ is not in $\mathcal{U}$.

Given any normal open subgroup $N$ of $\mathcal{G}$, the set $D$ consisting of those homomorphisms mapping the compact set $N$ to the open set $\mathcal{U}$ is precisely the set of homomorphisms factoring through $\mathcal{G} / N$, because any such homomorphism must send $N$ to the unique subgroup of $\mathcal{U}$. This set $D$ is therefore both open (in the compact-open topology) and closed (it is also the set of maps sending $N$ to the identity).

Every continuous homomorphism from $\mathcal{G}$ to $\mathrm{U}(n)$ factors through a finite quotient, as the image must be compact and because of what was proved above. As a result, $\operatorname{Hom}(\mathcal{G}, \mathrm{U}(n))$ is the colimit of spaces $\operatorname{Hom}(\mathcal{G} / N, \mathrm{U}(n))$ as $N$ varies over open normal subgroups of $\mathcal{G}$. Each of these spaces is an open and closed subset of the colimit. All of these are representation spaces of finite groups, and as a result they are compact.

Remark 21 In both cases, $\operatorname{Hom}(\mathcal{G}, \mathrm{U}(n))$ is a Hausdorff space and locally path connected.

The space $\operatorname{Hom}(\mathcal{G}, \mathrm{U}(n))$ has a continuous action of $\mathrm{U}(n)$ on it by conjugation. Define

$$
\operatorname{Rep}(\mathcal{G})=\coprod_{n \geq 0} \operatorname{Hom}(\mathcal{G}, \mathrm{U}(n)) / \mathrm{U}(n) .
$$

Because $\mathrm{U}(n)$ is path connected and compact, in the discrete and profinite cases $\operatorname{Rep}(\mathcal{G})$ is a Hausdorff locally path connected space. However, for a general group $\mathcal{G}$ there can be some point-set level pathology in this construction.

$\operatorname{Rep}(\mathcal{G})$ parameterizes isomorphism classes of continuous unitary representations of $\mathcal{G}$. It has a continuous augmentation map $\varepsilon: \operatorname{Rep}(\mathcal{G}) \rightarrow \mathbb{N}$ given by sending a representation to its dimension. Write $\mathcal{G}^{*}$ for the preimage of 1 under this map.

After making a choice of basis, there are continuous maps $\oplus: \mathrm{U}(n) \times \mathrm{U}(m) \rightarrow \mathrm{U}(n+m)$ and $\otimes: \mathrm{U}(n) \times \mathrm{U}(m) \rightarrow \mathrm{U}(n m)$ that induce continuous operations on $\operatorname{Rep}(\mathcal{G})$. These operations on $\operatorname{Rep}(\mathcal{G})$ are strictly commutative, associative, and distributive, and the augmentation $\varepsilon$ takes $\oplus$ to addition and $\otimes$ to multiplication. 
If $f: \mathcal{G} \rightarrow \mathcal{G}^{\prime}$ is a (continuous) group homomorphism, there is a continuous restriction map $f^{*}: \operatorname{Rep}\left(\mathcal{G}^{\prime}\right) \rightarrow \operatorname{Rep}(\mathcal{G})$ preserving $\oplus, \otimes$, and $\varepsilon$. $\operatorname{Rep}(-)$ is a contravariant functor to topological semirings, and $(-)^{*}$ is a contravariant functor to topological groups.

$\operatorname{Rep}(\mathcal{G})$ contains a subspace $\operatorname{Irr}(\mathcal{G})$ of irreducible representations. Tensoring an irreducible representation with a character yields a new irreducible representation, so $\operatorname{Irr}(\mathcal{G})$ is a $\mathcal{G}^{*}$-space. Because unitary representations have unique decompositions into irreducible representations, we have the following:

Proposition 22 The underlying set of $\operatorname{Rep}(\mathcal{G})$ is the free abelian monoid on the set $\operatorname{Irr}(\mathcal{G})$.

Corollary 23 If $\mathcal{G}$ is finite, $\operatorname{Rep}(\mathcal{G})$ is the free abelian topological monoid on the finite set $\operatorname{Irr}(\mathcal{G})$.

The following result, which allows us to examine the structure of $\operatorname{Rep}(\mathcal{G})$ explicitly for discrete nilpotent groups, first appeared in [9, Theorem 6.6].

Theorem 24 Suppose $\mathcal{G}$ is discrete, finitely generated, and nilpotent. There is a set $\left\{\rho_{\alpha}\right\}$ of representations of $\mathcal{G}$, all factoring through finite quotients, and finite subgroups $I_{\alpha}<\mathcal{G}^{*}$ such that

$$
\operatorname{Irr}(\mathcal{G}) \cong \coprod_{\alpha}\left(\mathcal{G}^{*} / I_{\alpha}\right) \cdot\left[\rho_{\alpha}\right]
$$

as a $\mathcal{G}^{*}$-space. Additionally, for any $n \in \mathbb{N}$ there are only finitely many $\rho_{\alpha}$ of dimension $n$.

Remark 25 The group $\mathcal{G}^{*}$ is a closed subgroup of $\prod U(1)$, and hence the spaces $\mathcal{G}^{*} / I_{\alpha}$ are finite unions of (possibly zero-dimensional) torii. Additionally, for any $\rho_{\alpha}$ the subgroup $I_{\alpha}$ consists of those characters $\psi$ such that $\psi \otimes_{\mathbb{C}} \rho_{\alpha} \cong \rho_{\alpha}$. This is necessarily finite: $\rho_{\alpha}$ factors through a finite quotient $\mathcal{G} / N$, so any such $\psi$ must also factor through $\mathcal{G} / N$. Therefore, $\psi$ can only range over the finite group of characters of $\mathcal{G} / N$.

Corollary 26 If $\mathcal{G}$ is discrete, finitely generated, and nilpotent, there is an isomorphism of $\mathcal{G}^{*}$-spaces

$$
\operatorname{Irr}(\mathcal{G}) \cong \mathcal{G}^{*} \times\left(\mathcal{G}^{\wedge}\right)^{*} \operatorname{Irr}\left(\mathcal{G}^{\wedge}\right),
$$

where $\mathcal{G}^{\wedge}$ is the profinite completion

$$
\lim _{[\mathcal{G}: N]<\infty} \mathcal{G} / N
$$


Proof As in Theorem 24, first write

$$
\operatorname{Irr}(\mathcal{G}) \cong \coprod_{\alpha} \mathcal{G}^{*} / I_{\alpha}\left[\rho_{\alpha}\right]
$$

where the $\rho_{\alpha}$ factor through finite quotients of $\mathcal{G}$. Therefore, we can assume that the $\rho_{\alpha}$ are actually elements of $\operatorname{Irr}\left(\mathcal{G}^{\wedge}\right)$. If $\rho_{\alpha}$ factors through a finite quotient of $\mathcal{G}$ and $\psi$ is a character of $\mathcal{G}$ such that $\psi \otimes \rho_{\alpha} \cong \rho_{\alpha}, \psi$ itself must factor through a finite quotient of $\mathcal{G}$. Therefore, the isotropy subgroup $I_{\alpha}$ of $\rho_{\alpha}$ is actually a subgroup of $\left(\mathcal{G}^{\wedge}\right)^{*}$.

Tensoring with characters of $\mathcal{G}^{\wedge}$ gives a map of $\left(\mathcal{G}^{\wedge}\right)^{*}$-sets

$$
\coprod_{\alpha}\left(\mathcal{G}^{\wedge}\right)^{*} / I_{\alpha} \cdot\left[\rho_{\alpha}\right] \rightarrow \operatorname{Irr}\left(\mathcal{G}^{\wedge}\right) .
$$

We claim that this map is an isomorphism, which proves the corollary. It is injective: composing with the map out to $\operatorname{Irr}(\mathcal{G})$ gives an injection

$$
\coprod\left(\mathcal{G}^{\wedge}\right)^{*} / I_{\alpha} \rightarrow \coprod \mathcal{G}^{*} / I_{\alpha}
$$

(The map $\left(\mathcal{G}^{\wedge}\right)^{*} \rightarrow \mathcal{G}^{*}$ is injective, because a character is determined by its values on the dense image of $\mathcal{G}$.) We now prove that the map of equation (5) is surjective.

Given an irreducible representation $\rho$ of $\mathcal{G}^{\wedge}$, its restriction to $\mathcal{G}$ is an irreducible representation $\rho$ of $\mathcal{G}$ on a vector space $W$. By the above decomposition of $\operatorname{Irr}(\mathcal{G})$, $\rho \cong \psi \otimes \rho_{\alpha}$ for some $\alpha$ : choose such an isomorphism $f: \mathbb{C} \otimes W_{\alpha} \rightarrow W$. Consider the $\mathcal{G}$-vector space $\operatorname{Hom}_{\mathbb{C}}\left(W_{\alpha}, W\right) \cong W_{\alpha}^{*} \otimes W$, where the dual and tensor are both taken over $\mathbb{C} . \mathcal{G}$ acts on $W$ and $W_{\alpha}$ through finite quotients, so the action of $\mathcal{G}$ on $\operatorname{Hom}_{\mathbb{C}}\left(W_{\alpha}, W\right)$ also factors through a finite quotient of $\mathcal{G}$. This vector space contains a map $f^{\prime}$, defined by $f^{\prime}(w)=f(1 \otimes w)$. Then $g$ acts on $f^{\prime}$ as follows:

$$
\begin{aligned}
\left(g f^{\prime}\right)(w) & =g \cdot f^{\prime}\left(g^{-1} w\right) \\
& =g \cdot f\left(1 \otimes g^{-1} w\right) \\
& =f\left(g \cdot\left(1 \otimes g^{-1} w\right)\right) \\
& =f(\psi(g) \otimes w) \\
& =\psi(g) f^{\prime}(w) .
\end{aligned}
$$

Therefore, the representation $\operatorname{Hom}_{\mathbb{C}}\left(W_{\alpha}, W\right)$ contains a 1-dimensional subspace $\left\langle f^{\prime}\right\rangle$ isomorphic to the representation $\psi$, so $\psi$ factors through a finite quotient of $\mathcal{G}$.

We are now in a position to prove the main result of this section. For a set $X$, let $\operatorname{Sym}^{k}(X)=X^{k} / \Sigma_{k}$ be the $k$-fold symmetric product of $X$, and for a based set $X$ 
let $\operatorname{Sym}^{\infty}(X)$ be the infinite symmetric product $\lim _{k} \operatorname{Sym}^{k}(X)$, where the limit is taken by adding additional copies of the basepoint.

Theorem 27 Suppose $\mathcal{G}$ is nilpotent, finitely topologically generated, and either profinite or discrete. Then $\operatorname{Rep}(\mathcal{G})$, the space of isomorphism classes of representations of $\mathcal{G}$, is homeomorphic to $\operatorname{Sym}^{\infty}\left(\operatorname{Irr}(\mathcal{G})_{+}\right)$, the free abelian topological monoid generated by the space $\operatorname{Irr}(\mathcal{G})$ of irreducible representations of $\mathcal{G}$.

Proof If $\mathcal{G}$ is profinite, then from Example 20 we can make the identification $\operatorname{Rep}(\mathcal{G})=$ $\operatorname{colim}_{N} \operatorname{Rep}(\mathcal{G} / N)$ as $N$ ranges over open normal subgroups of $\mathcal{G}$. The result follows from Corollary 23.

Now suppose that $\mathcal{G}$ is discrete. We have a continuous map of spaces $\operatorname{Irr}(\mathcal{G}) \rightarrow \operatorname{Rep}(\mathcal{G})$ that induces a continuous map of monoids $\operatorname{Sym}^{\infty}\left(\operatorname{Irr}(\mathcal{G})_{+}\right) \rightarrow \operatorname{Rep}(\mathcal{G})$. Because the underlying set of $\operatorname{Sym}^{\infty}\left(\operatorname{Irr}(\mathcal{G})_{+}\right)$is the free abelian monoid on $\operatorname{Irr}(\mathcal{G})$, this map is bijective.

We have also seen in Theorem 24 that $\operatorname{Irr}(\mathcal{G})$ is compact in fibers over $\mathbb{N}$. Because this is true for $\operatorname{Irr}(\mathcal{G})$, it is true for the symmetric products $\operatorname{Sym}^{k}(\operatorname{Irr}(\mathcal{G}))$, and hence true for the monoid $\operatorname{Sym}^{\infty}\left(\operatorname{Irr}(\mathcal{G})_{+}\right) \cong \coprod \operatorname{Sym}^{k}(\operatorname{Irr}(\mathcal{G}))$. The result follows because $\operatorname{Rep}(\mathcal{G})$ is Hausdorff.

\section{Deformation representation ring spectra}

Let $\mathcal{G}$ be a topological group. From this group $\mathcal{G}$, we will now construct a deformation representation ring spectrum $R[\mathcal{G}]$ by analogy with the construction for a finite group. The constructions here are a modification of those that yield a "deformation" $K$-theory spectrum (see [6]), and the homotopy groups of $R[\mathcal{G}]$ will be shown in forthcoming work to be involved in a spectral sequence that computes the homotopy groups of deformation $K$-theory.

The space $\operatorname{Rep}(\mathcal{G})$ of Section 3 is an abelian topological monoid under $\oplus$, so we can apply an iterated classifying space construction to it; the result is a (naïve pre-) spectrum whose $k$ 'th space is $\left\{B^{(k)} \operatorname{Rep}(\mathcal{G})\right\}$. The honestly commutative product structure induced by $\otimes$ on $\operatorname{Rep}(\mathcal{G})$ will give this object the structure of an $E_{\infty}$-ring spectrum.

To make this construction more rigid, we will make use of the $\Gamma$-spaces of Segal [15], using the smash product defined by Lydakis in [10]. These are a model of connective spectra particularly suited for this kind of application. See Schwede [13] for an exposition of the homotopy theory of these objects; in particular, the appendix contains 
an introduction to the "topological $\Gamma$-spaces" that we will be using. The relationship of these objects with other structured categories of spectra can be found in MandellMay-Schwede-Shipley [11]. (In that article, $\Gamma$-spaces are referred to as $\mathcal{F}$-spaces.) The structured ring and module objects created in this section can be converted into symmetric spectra using a Quillen equivalence; by [11, Theorems $0.1,0.3]$, the resulting objects are commutative monoid objects in symmetric spectra. Similarly, applying [14, Theorem 5.1], the resulting monoids in symmetric spectra can be converted using a Quillen equivalence to commutative $\mathbb{S}$-algebras.

We first recall some definitions and properties of $\Gamma$-spaces.

Definition $28 \Gamma^{o}$ is the category of finite based sets.

Definition 29 A $\Gamma$-space is a functor $M: \Gamma^{o} \rightarrow$ Top $_{*}$ from finite based sets to based spaces such that $M(*)=*$. A map of $\Gamma$-spaces is a natural transformation of functors.

Definition 30 The sphere spectrum $\Gamma$-space is defined by $\mathbb{S}(X)=X$.

Definition 31 If $M$ is a $\Gamma$-space and $K$ is a pointed space, define the tensor product $\Gamma$-space by

$$
(M \otimes K)(X)=M(X) \wedge K
$$

Definition 32 If $M$ and $N$ are $\Gamma$-spaces, the smash product $\Gamma$-space is defined as follows: for $Z \in \Gamma^{o}$,

$$
M \wedge N(Z)=\operatorname{colim}_{X \wedge Y \rightarrow Z} M(X) \wedge N(Y)
$$

The object $\mathbb{S} \otimes K$ is the suspension spectrum $\Gamma$-space associated to $K$.

Left for exercises are proofs of the standard facts: the smash product is a symmetric monoidal product on $\Gamma$-spaces with unit $\mathbb{S}$, and there are identifications

$$
M \wedge(N \otimes K) \cong(M \wedge N) \otimes K
$$

Remark 33 This definition of the smash product is a left Kan extension of the functor $M \wedge N: \Gamma^{o} \times \Gamma^{o} \rightarrow \mathbf{T o p}_{*}$ along the functor $\wedge: \Gamma^{o} \times \Gamma^{o} \rightarrow \Gamma^{o}$. Therefore, in order to define a map from $M \wedge N$ to $P$ it suffices to define natural maps $M(X) \wedge N(Y) \rightarrow$ $P(X \wedge Y)$. 
A symmetric spectrum is constructed from a $\Gamma$-space $M$ in the following way. First, we prolong the functor $M$ to a functor on finite pointed simplicial sets by applying $M$ levelwise and taking geometric realization. Second, we note that there is a natural assembly map as follows for $X \in \Gamma^{o}$ :

$$
X \wedge M(Y) \cong \bigvee_{X} M(Y) \rightarrow M\left(\bigvee_{X} Y\right) \cong M(X \wedge Y)
$$

This can also be prolonged to the case when $X$ is the geometric realization of a finite simplicial set. The resulting symmetric spectrum is the sequence of spaces $M\left(\left(S^{1}\right)^{\wedge n}\right)$, with structure maps coming from the symmetric group action on the smash product and the structure map

$$
S^{1} \wedge M\left(\left(S^{1}\right)^{\wedge n}\right) \rightarrow M\left(\left(S^{1}\right)^{\wedge(n+1)}\right) .
$$

This association respects the symmetric monoidal structure. We will denote this spectrum by $\mathcal{S} p(M)$.

Definition 34 If $A$ is an abelian topological monoid, define a $\Gamma$-space $\tilde{A}$ as follows. For $Z \in \Gamma^{o}$, define

$$
\widetilde{A}(Z)=\operatorname{Map}_{*}(Z, A),
$$

where the unit of $A$ is regarded as its basepoint. For $f: Z \rightarrow Z^{\prime}$ and $\alpha \in \operatorname{Map}_{*}(Z, A)$, define

$$
\left(f_{*} \alpha\right)\left(z^{\prime}\right)=\sum_{f(z)=z^{\prime}} \alpha(z)
$$

Proposition 35 A continuous bilinear pairing $\mu: A \times B \rightarrow C$ of abelian topological monoids naturally gives rise to a map $\tilde{\mu}: \widetilde{A} \wedge \widetilde{B} \rightarrow \widetilde{C}$ of $\Gamma$-spaces.

Proof This is defined in the obvious way. We define $\tilde{\mu}: \widetilde{A}(X) \wedge \widetilde{B}(Y) \rightarrow \widetilde{C}(X \wedge Y)$ by

$$
\tilde{\mu}(\alpha \wedge \beta)(x \wedge y)=\mu(\alpha(x), \beta(y)) .
$$


That this is natural in $X$ and $Y$ follows from the bilinearity of $\mu$ as follows. Suppose $f: X \rightarrow X^{\prime}$. Then

$$
\begin{aligned}
\tilde{\mu}\left(f_{*} \alpha \wedge \beta\right)\left(x^{\prime} \wedge y\right) & =\mu\left(f_{*} \alpha\left(x^{\prime}\right), \beta(y)\right) \\
& =\mu\left(\sum_{f(x)=x^{\prime}} \alpha(x), \beta(y)\right) \\
& =\sum_{f(x)=x^{\prime}} \tilde{\mu}(\alpha \wedge \beta)(x \wedge y) \\
& =(f \wedge 1)_{*} \tilde{\mu}(\alpha \wedge \beta)(x \wedge y) .
\end{aligned}
$$

The naturality in $Y$ is symmetric.

Remark 36 Any abelian topological monoid $A$ has a unique bilinear pairing $\mathbb{N} \times A \rightarrow$ $A$ taking $1 \times a$ to $a$. If $R$ is a topological semiring (an object with addition and multiplication operations, but no additive inverses), there is a unique map $\mathbb{N} \rightarrow R$ respecting the multiplication. As a result, in $\Gamma$-spaces $\widetilde{\mathbb{N}}$ is a commutative monoid, any $\widetilde{A}$ is a module over $\widetilde{\mathbb{N}}$, and maps of topological monoids realize to maps of $\widetilde{\mathbb{N}}$-modules. In other words, the functor $\widetilde{(-)}$ has range in $\widetilde{\mathbb{N}}$-modules.

Any $\widetilde{R}$ comes equipped with a multiplicative map $\widetilde{\mathbb{N}} \rightarrow \widetilde{R}$. Additionally, if $A \times B \rightarrow C$ is a bilinear pairing, the map $\widetilde{A} \wedge \widetilde{B} \rightarrow \widetilde{C}$ respects the $\widetilde{\mathbb{N}}$-action.

We now define the deformation representation ring functor.

Definition 37 The deformation representation ring $R[\mathcal{G}]$ is the spectrum $\mathcal{S} p(\widetilde{\operatorname{Rep}(\mathcal{G})})$.

For any abelian topological monoid $A$, the $\Gamma$-space $\tilde{A}$ is special (see [13]), meaning that the map

$$
\widetilde{A}(X \vee Y) \rightarrow \widetilde{A}(X) \times \widetilde{A}(Y)
$$

is an equivalence for all $X, Y$. (In this case, it is actually a homeomorphism.) As a result, the infinite loop space $\Omega^{\infty} \mathcal{S} p \tilde{A}$ can be analyzed. The adjoint of the assembly map $\widetilde{A}\left(S^{0}\right) \rightarrow \Omega \widetilde{A}\left(S^{1}\right)$ is the homotopy group completion map $A \rightarrow \Omega B A$ of the monoid $A$. Since $\left(\tilde{A} \otimes S^{n}\right)\left(S^{0}\right)$ is connected, by [15, Proposition 1.4] the map $\tilde{A}\left(S^{n}\right) \rightarrow$ $\Omega \widetilde{A}\left(S^{n} \wedge S^{1}\right)$ is a homotopy equivalence for $n>0$, implying that $\Omega^{n} \widetilde{A}\left(S^{n}\right) \simeq \Omega B A$ for $n>0$. Taking limits shows that $\Omega^{\infty} \mathcal{S} p(\tilde{A}) \simeq \Omega B A$.

$R[-]$ is a contravariant functor from groups to commutative monoids in symmetric spectra. In fact, $\Omega^{\infty} \mathcal{S} p(\tilde{\mathbb{N}}) \simeq \mathbb{Z}$, so the spectrum $\mathcal{S} p(\tilde{\mathbb{N}})$ is an Eilenberg-MacLane spectrum equivalent to $\mathbb{H} \mathbb{Z}$. (We will show this explicitly in Proposition 38.) The 
functor $R[-]$ takes values in $\mathbb{H} \mathbb{Z}$-algebras. There is also a natural augmentation $\varepsilon: R[\mathcal{G}] \rightarrow \mathbb{H} \mathbb{Z}$ coming from the augmentation $\operatorname{Rep}(\mathcal{G}) \rightarrow \mathbb{N}$.

Proposition 38 Suppose $\mathcal{G}$ is finitely topologically generated, nilpotent, and either discrete or profinite. Then $R[\mathcal{G}]$ is equivalent to $\mathbb{Z} \mathbb{Z} \wedge \Sigma_{+}^{\infty} \operatorname{Irr}(\mathcal{G})$ as a module over the group ring spectrum $\mathbb{Z} \mathbb{Z} \wedge \Sigma_{+}^{\infty} \mathcal{G}^{*}$.

As a result, $\pi_{*}(R[\mathcal{G}]) \cong H_{*}(\operatorname{Irr}(\mathcal{G}))$.

Proof We have a natural identification of spaces $\widetilde{\operatorname{Rep}(\mathcal{G})}\left(S^{0}\right)=\operatorname{Rep}(\mathcal{G})$, and so there are natural maps of pointed spaces:

$$
\left(\mathcal{G}^{*}\right)_{+} \rightarrow \operatorname{Irr}(\mathcal{G})_{+} \rightarrow R[\mathcal{G}]\left(S^{0}\right) .
$$

For $X \in \Gamma^{o}$, taking a wedge over the points of $X$ gives natural maps as follows:

$$
X \wedge\left(\mathcal{G}^{*}\right)_{+} \rightarrow X \wedge \operatorname{Irr}(\mathcal{G})_{+} \rightarrow X \wedge \widehat{\operatorname{Rep}(\mathcal{G})}\left(S^{0}\right) \rightarrow \widetilde{\operatorname{Rep}(\mathcal{G})}(X)
$$

The right-hand map is the assembly map.

Naturality implies that these give rise to maps

$$
\mathbb{S} \otimes\left(\mathcal{G}^{*}\right)_{+} \rightarrow \mathbb{S} \otimes \operatorname{Irr}(\mathcal{G})_{+} \rightarrow \widetilde{\operatorname{Rep}(\mathcal{G})}
$$

of $\Gamma$-spaces. Additionally, for $X, Y \in \Gamma^{o}$ there is a commutative diagram as follows.

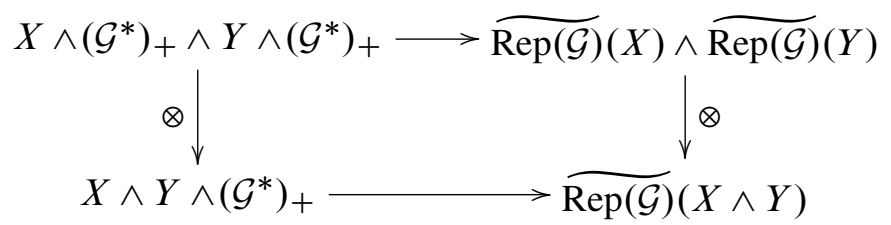

This is natural in $X$ and $Y$, and as a result it translates into the following diagram of $\Gamma$-spaces.

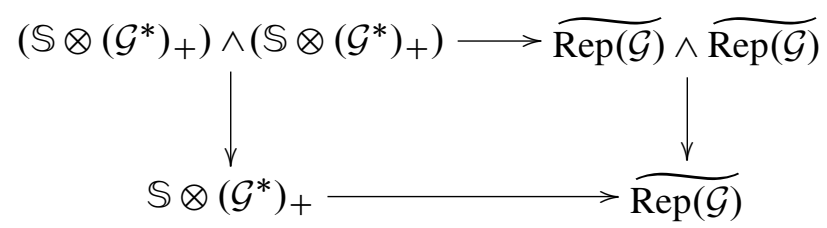

This gives $\widetilde{\operatorname{Rep}(\mathcal{G})}$ a natural structure of an algebra over the group ring $\Gamma$-space $\mathbb{S}\left[\mathcal{G}^{*}\right]=\mathbb{S} \otimes\left(\mathcal{G}^{*}\right)_{+} . \widetilde{\operatorname{Rep}(\mathcal{G})}$ is already a commutative $\widetilde{\mathbb{N}}$-algebra, so $\widetilde{\operatorname{Rep}(\mathcal{G})}$ is naturally an algebra over $\widetilde{\mathbb{N}} \wedge \mathbb{S}\left[\mathcal{G}^{*}\right] \cong \widetilde{\mathbb{N}}\left[\mathcal{G}^{*}\right]$.

The map $\mathbb{S} \otimes \operatorname{Irr}(\mathcal{G})_{+} \rightarrow \widetilde{\operatorname{Rep}(\mathcal{G})}$ also extends naturally to a map $\tilde{\mathbb{N}} \otimes \operatorname{Irr}(\mathcal{G})_{+} \rightarrow \widetilde{\operatorname{Rep}(\mathcal{G})}$. Similarly, we can give $\widetilde{\mathbb{N}} \otimes \operatorname{Irr}(\mathcal{G})_{+}$the structure of a module over $\widetilde{\mathbb{N}}\left[\mathcal{G}^{*}\right]$ in a natural 
way making the map $\tilde{\mathbb{N}} \otimes \operatorname{Irr}(\mathcal{G})_{+} \rightarrow \widetilde{\operatorname{Rep}(\mathcal{G})}$ a map of modules over $\widetilde{\mathbb{N}}\left[\mathcal{G}^{*}\right]$. We claim that this map gives a stable equivalence of spectra. In fact, this follows from Theorem 27 and a generalized Dold-Thom theorem, which states that the spectrum $\mathcal{S} p\left(\widehat{\operatorname{Sym}^{\infty}(M)}\right)$ is equivalent to $\mathbb{H} \mathbb{Z} \wedge \Sigma_{+}^{\infty} M$ for any space $M$ of the homotopy type of a CW-complex. The natural map inducing the Dold-Thom equivalence

$$
\widetilde{\mathbb{N}} \otimes \operatorname{Irr}(\mathcal{G})_{+} \rightarrow \widehat{\operatorname{Sym}^{\infty}(\operatorname{Irr}(\mathcal{G}))}
$$

upon applying $\mathcal{S} p$, becomes an equivalence

$$
\mathbb{H} \mathbb{Z} \wedge \Sigma_{+}^{\infty} \operatorname{Irr}(\mathcal{G})_{+} \rightarrow R[\mathcal{G}] .
$$

Since this was a map of modules over the $\Gamma$-space $\widetilde{\mathbb{N}}\left[\mathcal{G}^{*}\right]$, applying the monoidal functor $\mathcal{S} p$ gives a map of modules over the group ring spectrum $\mathbb{R} \mathbb{Z} \wedge \Sigma_{+}^{\infty} \mathcal{G}^{*}$.

Corollary 39 When $\mathcal{G}$ is finite, $R[\mathcal{G}]$ is an Eilenberg-MacLane spectrum associated to the ordinary complex representation ring of $\mathcal{G}$, and when $\mathcal{G}$ is profinite, $R[\mathcal{G}] \cong$ $\operatorname{colim}_{[\mathcal{G}: N]<\infty} R[\mathcal{G} / N]$ is also an Eilenberg-MacLane spectrum.

Proof In these cases, $\operatorname{Irr}(\mathcal{G})$ is discrete.

\section{$5 \quad R[\mathcal{G}]$ and Bousfield localization}

Throughout this section $\mathcal{G}$ is a finitely generated discrete nilpotent group, having profinite completion $\mathcal{G}^{\wedge}$ and pro- $p$-completions $\mathcal{G}_{p}^{\wedge}$. Let $\ell$ denote a prime at which completions of spectra will be taken. The "localization" of an $R$-module $M$ will always refer to Bousfield localization with respect to $\mathbb{H F}_{\ell}$, where $\mathbb{M} \mathbb{F}_{\ell}$ has an explicit module structure over some base $\mathbb{S}$-algebra $R$. (In this section, we will make use of $\mathbb{S}$-modules as our base category of spectra, unless otherwise specified. As described in the beginning of Section 4, the ring objects of the previous section will be implicitly converted into $\mathbb{S}$-algebras, retaining their names.)

There is an augmentation map $\varepsilon: R[\mathcal{G}] \rightarrow \mathbb{G} \ell$ given by taking a representation to its dimension $\bmod \ell$. This is a map of commutative $\mathbb{S}$-algebras.

In this section we will proceed with the proof of Theorem 2 by breaking it up into the two following statements.

Proposition 40 The map $R\left[\mathcal{G}^{\wedge}\right] \rightarrow R[\mathcal{G}]$ is a multiplicative $\mathbb{H} \mathbb{F}_{\ell}$-equivalence, and the map $R\left[\mathcal{G}_{\ell}^{\wedge}\right] \rightarrow R\left[\mathcal{G}^{\wedge}\right]$ is a multiplicative $\mathbb{H} \mathbb{F}_{\ell}$-equivalence. 
Proof The proof of the first statement is organized by progressively proving it for more general cases.

The case when $\mathcal{G}$ is a finite abelian group follows because $\mathcal{G}^{\wedge} \cong \mathcal{G}$. Section 5.1 carries out the proof in the special case $\mathcal{G}=\mathbb{Z}$.

In Section 5.2, we prove Lemma 43, which shows that if $R[G] \rightarrow R\left[G^{\prime}\right]$ is a multiplicative $\mathbb{H} \mathbb{F}_{\ell}$-equivalence, so is $R[G \times H] \rightarrow R\left[G^{\prime} \times H\right]$. If $\mathcal{G}$ is a finitely generated abelian group, Lemma 43 then allows us to prove the theorem by using the existence of a decomposition $\mathcal{G} \cong \mathbb{Z}^{d} \oplus A$, where $A$ is a finite abelian group.

In Section 5.3, we finish the proof of the first statement by making use of Proposition 12 and the structure of $R[\mathcal{G}]$ and $R\left[\mathcal{G}^{\wedge}\right]$ as algebras over $R\left[\left(\mathcal{G}^{\wedge}\right)_{\mathrm{ab}}\right]$.

If $\mathcal{G}$ is nilpotent, then by definition some iterated bracket map

$$
[-,[-,[\cdots]]]: \mathcal{G}^{r} \rightarrow \mathcal{G}
$$

is trivial. The image of $\mathcal{G}^{r}$ is dense in $\left(\mathcal{G}^{\wedge}\right)^{r}$, and so the iterated bracket map is also trivial for $\mathcal{G}^{\wedge}$. Hence $\mathcal{G}^{\wedge}$ is nilpotent.

Using this, the proof of the second statement is the content of Section 5.4, Corollary 45 .

\subsection{The case of $\mathcal{G}=\mathbb{Z}$}

First, we compute the representation rings of the groups $\mathbb{Z}$, its profinite completion $\hat{\mathbb{Z}}$, and the $p$-adic integers $\mathbb{Z}_{p}$, where $p$ is a prime. By the results in Section 4 , it suffices to compute the spaces of irreducible representations.

Let $\mu_{\infty}$ be the group of roots of unity in $\mathbb{C}$, and let $\mu_{p} \infty$ be the subgroup consisting of elements whose order is a power of $p$.

Lemma 41 The maps $\operatorname{Irr}\left(\mathbb{Z}_{p}\right) \rightarrow \operatorname{Irr}(\hat{\mathbb{Z}}) \rightarrow \operatorname{Irr}(\mathbb{Z})$ are the maps

$$
\mu_{p} \infty \mu_{\infty} \rightarrow S^{1}
$$

where $\mu_{\infty}$ and $\mu_{p^{\infty}}$ have the discrete topology.

Proof Any unitary representation of an abelian group is diagonalizable, so the spaces of irreducible representations must be precisely the spaces of 1-dimensional representations.

Proposition 42 The map $R[\widehat{\mathbb{Z}}] \rightarrow R[\mathbb{Z}]$ is a multiplicative $\mathbb{H} \mathbb{F}_{\ell}$-equivalence. 
Proof We need to show that the following map is an equivalence:

$$
\mathbb{H} \mathbb{F}_{\ell} \rightarrow \mathfrak{H} \mathbb{F}_{\ell} \underset{R[\widehat{\mathbb{Z}}]}{\wedge} R[\mathbb{Z}]
$$

Choose a sequence of primitive roots of unity $\zeta_{m}$ of $\mu_{m}$ such that $\zeta_{m}=\left(\zeta_{d m}\right)^{d}$. We identify $R[\mathbb{Z}]$ with the group ring spectrum $\mathbb{H} \mathbb{Z}\left[S^{1}\right]$.

By [7, Proposition IV.7.5], for a $\mathbb{S}$-algebra $R$ with right module $M$ and left module $N$, there is an equivalence of the geometric realization of the bar complex $B(M, R, N)$ with $M \wedge_{R} N$. If $M$ and $N$ are modules over a directed system of algebras $\left\{R_{\alpha}\right\}$ with homotopy colimit equivalent to $R$, taking homotopy colimits implies that

$$
M \underset{R}{\wedge} N \simeq \operatorname{hocolim} M \underset{R_{\alpha}}{\wedge} N .
$$

(If the system is not directed, the homotopy colimit as $\mathbb{S}$-algebras does not necessarily coincide with the homotopy colimit as $\mathbb{S}$-modules.) Therefore, the smash product commutes with directed homotopy colimits in the $\mathbb{S}$-algebra variable.

The map hocolim $_{m} \mathbb{H} \mathbb{Z}\left[\mu_{m}\right] \rightarrow R[\hat{\mathbb{Z}}]$ is an equivalence. It therefore suffices to show that the map

$$
\mathbb{H} \mathbb{F}_{\ell} \rightarrow \operatorname{hocolim} \mathbb{M} \mathbb{F}_{\ell} \underset{\mathbb{Z}\left[\mu_{m}\right]}{\wedge} \mathbb{H} \mathbb{Z}\left[S^{1}\right]
$$

is an equivalence.

As a $\mu_{m}$-space, $S^{1}$ is homeomorphic to the homotopy colimit of the diagram

$$
\mu_{m} \stackrel{\zeta_{m}}{\rightrightarrows} \mu_{m}
$$

Applying $\mathbb{H} \mathbb{Z} \wedge \Sigma_{+}^{\infty}$, this leads to the exact triangle of $\mathbb{H} \mathbb{Z}\left[\mu_{m}\right]$-modules

$$
\mathbb{H} \mathbb{Z}\left[\mu_{m}\right] \stackrel{\zeta_{m}-1}{\longrightarrow} \mathbb{H} \mathbb{Z}\left[\mu_{m}\right] \rightarrow \mathbb{H} \mathbb{Z}\left[S^{1}\right] .
$$

Recall that the augmentation $\mathbb{Z}\left[\mu_{m}\right] \rightarrow \mathbb{F}_{\ell}$ is the unique ring map taking the elements of $\mu_{m}$ to 1 . Applying $\mathbb{A} \mathbb{F} \ell \wedge_{\mathbb{H} \mathbb{Z}\left[\mu_{m}\right]}-$ to the above exact triangle gives the exact triangle

$$
\mathbb{H} \mathbb{F}_{\ell} \stackrel{0}{\rightarrow} \mathbb{M} \mathbb{F}_{\ell} \rightarrow \mathbb{H} \mathbb{F}_{\ell} \underset{\mathbb{H} \mathbb{Z}\left[\mu_{m}\right]}{\wedge} \mathbb{M} \mathbb{Z}\left[S^{1}\right]
$$

The inclusion $\iota: \mu_{m} \rightarrow \mu_{d m}$ induces the following map of exact triangles.

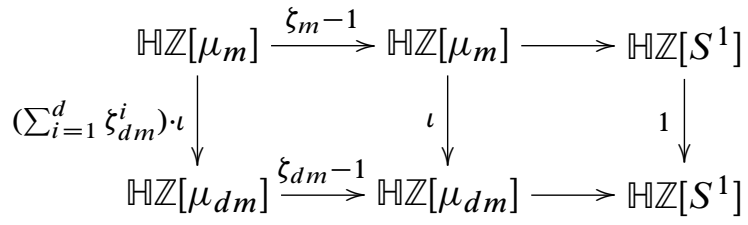


As a result, after smashing with $\mathbb{H} \mathbb{F}_{\ell}$, there is the following induced map of exact triangles.

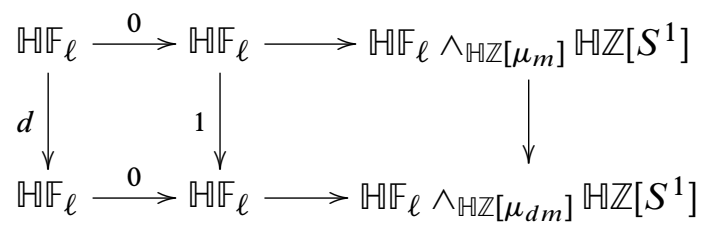

Taking homotopy colimits in $m$, we find that the map

$$
\mathbb{H} \mathbb{F}_{\ell} \rightarrow \operatorname{hocolim} \mathbb{M} \mathbb{F}_{\ell} \underset{\mathbb{Z}\left[\mu_{m}\right]}{\wedge} \mathbb{H} \mathbb{Z}\left[S^{1}\right]
$$

is an equivalence, as desired.

\subsection{The case of $\mathcal{G}$ finitely generated abelian}

If $\mathcal{G}$ is a finitely generated abelian group, we will show in this section that the map $R\left[\mathcal{G}^{\wedge}\right] \rightarrow R[\mathcal{G}]$ induces an isomorphism after localization.

If $\mathcal{G}$ is cyclic, then either $\mathcal{G}$ is finite or $\mathcal{G}=\mathbb{Z}$, and both of these cases have already been proven. The case of a finitely generated abelian group follows immediately from the following lemma.

Lemma 43 If $G, G^{\prime}$, and $H$ are nilpotent groups and a map $G \rightarrow G^{\prime}$ induces a multiplicative $\mathbb{M} \mathbb{F}_{\ell}$-equivalence $R\left[G^{\prime}\right] \rightarrow R[G]$, then so does the map $G \times H \rightarrow G^{\prime} \times H$.

Proof We first note that from Theorem $27, R[G \times H] \cong R[G] \wedge_{\mathbb{H} \mathbb{Z}} R[H]$ because $\operatorname{Irr}(G \times H) \cong \operatorname{Irr}(G) \times \operatorname{Irr}(H)$.

We need to show that the map $R[G \times H] \rightarrow R\left[G^{\prime} \times H\right]$ induces an isomorphism after applying $\mathbb{H F}_{\ell} \wedge R[G \times H](-)$.

As $R[G] \wedge_{\mathbb{H} \mathbb{Z}} R[H]$-modules, we have that

$$
\mathbb{H} \mathbb{F}_{\ell} \simeq \mathbb{H} \mathbb{F}_{\ell} \underset{\mathbb{H} \mathbb{Z}}{\wedge} \mathbb{H} \mathbb{Z}
$$

and

$$
R\left[G^{\prime} \times H\right] \simeq R\left[G^{\prime}\right] \stackrel{\wedge}{\wedge} R[H]
$$

By carrying out the proof of [7, Proposition III.3.10], with base ring $\mathbb{M} \mathbb{Z}$ rather than $\mathbb{S}$, we get a smash product identity

$$
(A \underset{\leftrightarrow \mathbb{Z}}{\wedge} B) \underset{\left(R \wedge \hat{\mathbb{Z}} R^{\prime}\right)}{\wedge}(C \underset{\leftrightarrow \leftrightarrow \mathbb{Z}}{\wedge} D) \cong(A \underset{R}{\wedge} C) \stackrel{\wedge}{\wedge}\left(B \underset{R^{\prime}}{\wedge} D\right) .
$$


This allows us to obtain the following equivalences:

$$
\begin{aligned}
& \mathbb{H} \mathbb{F}_{\ell} \underset{R[G \times H]}{\wedge} R\left[G^{\prime} \times H\right] \simeq\left(\mathbb{M} \mathbb{F}_{\ell} \underset{R[G]}{\wedge} R\left[G^{\prime}\right]\right) \underset{\mathbb{H Z}}{\wedge}(\mathbb{M} \mathbb{Z} \underset{R[H]}{\wedge} R[H]) \\
& \simeq\left(\mathbb{H F}_{\ell} \underset{R[G]}{\wedge} R[G]\right) \underset{\mathbb{H} \mathbb{Z}}{\wedge}(\mathbb{M} \mathbb{Z} \underset{R[H]}{\wedge} R[H]) \\
& \simeq \mathbb{M} \mathbb{F}_{\ell} \underset{R[G \times H]}{\wedge}(R[G] \stackrel{\wedge}{\wedge} R[H]) \\
& \simeq \mathbb{U} \mathbb{F}_{\ell} \text {. }
\end{aligned}
$$

\subsection{The case of a general nilpotent $\mathcal{G}$}

We now complete the proof of the first statement of Proposition 40. The general case of a discrete finitely generated nilpotent group $\mathcal{G}$ will follow from the machinery of Section 2 and our explicit description of the space of irreducible representations of a nilpotent group.

Let $k$ be $R\left[\left(\mathcal{G}^{\wedge}\right)^{*}\right]$, which is an Eilenberg-MacLane spectrum associated to the representation ring of $\left(\mathcal{G}^{\wedge}\right)_{\mathrm{ab}}$ by Corollary 39. The restriction map $k \rightarrow R\left[\mathcal{G}^{\wedge}\right]$ gives $R\left[\mathcal{G}^{\wedge}\right]$ and $R[\mathcal{G}]$ the structure of $k$-algebras.

By Proposition 12, it suffices to show that there is an equivalence

$$
\mathbb{M} \mathbb{F}_{\ell} \underset{k}{\wedge} R\left[\mathcal{G}^{\wedge}\right] \rightarrow \mathbb{W} \mathbb{F}_{\ell} \underset{k}{\wedge} R[\mathcal{G}]
$$

From Corollary 26 we have that

$$
\operatorname{Irr}(\mathcal{G}) \cong \mathcal{G}^{*} \underset{\left(\mathcal{G}^{\wedge}\right)^{*}}{\times} \operatorname{Irr}\left(\mathcal{G}^{\wedge}\right) .
$$

By Theorem 24, the space $\operatorname{Irr}\left(\mathcal{G}^{\wedge}\right)$ breaks up as a coproduct of orbits of $\left(\mathcal{G}^{\wedge}\right)^{*}$ with finite stabilizers. If $K$ is a finite subgroup of $\left(\mathcal{G}^{\wedge}\right)^{*}$, then $K$ acting freely on $\mathcal{G}^{*}$ implies that there is a weak equivalence of $\mathcal{G}^{*} / K$ with the realization of the bar construction $B\left(\mathcal{G}^{*}, K, *\right)$. The suspension spectrum functor is strong symmetric monoidal by [7, Proposition II.1.2], so applying $\Sigma_{+}^{\infty}$ gives an equivalence

$$
\begin{aligned}
\Sigma_{+}^{\infty}\left(\mathcal{G}^{*} / K\right) & \simeq B\left(\Sigma_{+}^{\infty} \mathcal{G}^{*}, \Sigma_{+}^{\infty} K, \mathbb{S}\right) \\
& \simeq \Sigma_{+}^{\infty} \mathcal{G}^{*}{ }_{\Sigma_{+}^{\infty} K} \mathbb{S} .
\end{aligned}
$$

Similarly,

$$
\Sigma_{+}^{\infty}\left(\mathcal{G}^{\wedge}\right)^{*} / K \simeq \Sigma_{+}^{\infty}\left(\mathcal{G}^{\wedge}\right)^{*} \underset{\Sigma_{+}^{\infty} K}{\wedge} \mathbb{S} .
$$


The map $\Sigma_{+}^{\infty}\left(\mathcal{G}^{\wedge}\right)^{*} / K \rightarrow \Sigma_{+}^{\infty} \mathcal{G}^{*} / K$ then induces an equivalence

$$
\Sigma_{+}^{\infty} \mathcal{G}^{*} \underset{\Sigma_{+}^{\infty}\left(\mathcal{G}^{\wedge}\right)^{*}}{\wedge} \Sigma_{+}^{\infty}\left(\mathcal{G}^{\wedge}\right)^{*} / K \rightarrow \Sigma_{+}^{\infty} \mathcal{G}^{*} / K .
$$

Smashing with $\mathbb{H} \mathbb{Z}$ and taking the union over all orbits, we find that

$$
\mathfrak{H} \mathbb{Z} \wedge \Sigma_{+}^{\infty} \operatorname{Irr}(\mathcal{G}) \simeq\left(\mathbb{H} \mathbb{Z} \wedge \Sigma_{+}^{\infty} \mathcal{G}^{*}\right) \underset{k}{\wedge}\left(\mathbb{H} \mathbb{Z} \wedge \Sigma_{+}^{\infty} \operatorname{Irr}\left(\mathcal{G}^{\wedge}\right)\right) .
$$

Applying $\mathbb{H F}_{\ell} \wedge_{k}-$ to this equivalence gives the following new equivalences.

$$
\begin{aligned}
& \mathbb{M} \mathbb{F}_{\ell}{\underset{k}{k}}_{R}[\mathcal{G}] \simeq \mathbb{M} \mathbb{F}_{\ell} \hat{k}^{\wedge}\left(\mathbb{M} \mathbb{Z} \wedge \Sigma_{+}^{\infty} \operatorname{Irr}(\mathcal{G})\right)
\end{aligned}
$$

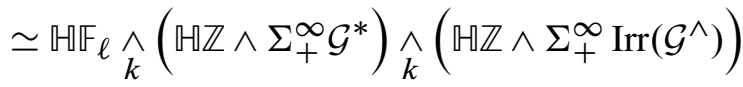

$$
\begin{aligned}
& \simeq\left(\mathbb{H} \mathbb{F}_{\ell} \underset{k}{\wedge} R\left[\mathcal{G}_{\mathrm{ab}}\right]\right) \underset{k}{\wedge}\left(\mathbb{H} \mathbb{Z} \wedge \Sigma_{+}^{\infty} \operatorname{Irr}\left(\mathcal{G}^{\wedge}\right)\right) \\
& \simeq \mathbb{H} \mathbb{F}_{\ell} \underset{k}{\wedge} R\left[\mathcal{G}^{\wedge}\right]
\end{aligned}
$$

(The equivalence $\mathbb{M} \mathbb{F}_{\ell} \rightarrow \mathbb{M} \mathbb{F}_{\ell} \wedge_{k} R\left[\mathcal{G}_{\mathrm{ab}}\right]$ follows from the results of Section 5.2, applied to $\mathcal{G}_{\mathrm{ab}}$.)

\subsection{Localizations for non- $\ell$-groups}

The result of this section is that the representation rings of profinite groups with no $\ell$-primary part become trivial upon localization. The first result of this section does not depend on $\mathcal{G}$ being nilpotent.

Lemma 44 Suppose $\mathcal{G}$ is a profinite group whose finite quotients all have order prime to $\ell$. Then the augmentation map $R[\mathcal{G}] \rightarrow \mathbb{H} \mathbb{Z}$ is a multiplicative $\mathbb{H} \mathbb{F}_{\ell}$-equivalence.

Proof Note that $R[\mathcal{G}] \simeq$ hocolim $R\left[\mathcal{G}_{\beta}\right]$, where the $\mathcal{G}_{\beta}$ form the directed system of finite quotients of $\mathcal{G}$. As a result, it is therefore necessary and sufficient to prove that the map $\mathbb{U} \mathbb{F}_{\ell} \rightarrow \mathbb{G} \mathbb{F}_{\ell} \wedge R[\mathcal{G}] \mathbb{Z} \mathbb{Z}$ is an equivalence when $\mathcal{G}$ is a finite prime-to$\ell$-group. Because $R[\mathcal{G}] \simeq \mathbb{U} R$ for a ring $R$, the derived category of $R$-modules, with derived tensor product, is equivalent to the category of modules over $R[\mathcal{G}]$, by [7, Theorem IV.2.4]. Therefore, it suffices to show that the map $R \rightarrow \mathbb{Z}$ induces an isomorphism

$$
\mathbb{F}_{\ell} \rightarrow \mathbb{F}_{\ell} \otimes_{R}^{\mathbb{Z}} \mathbb{Z}
$$

$R$ is projective over $\mathbb{Z}$, so we can compute these Tor groups using the bar complex

$$
\cdots \rightarrow \mathbb{F}_{\ell} \otimes R \otimes R \otimes \mathbb{Z} \rightarrow \mathbb{F}_{\ell} \otimes R \otimes \mathbb{Z} \rightarrow \mathbb{F}_{\ell} \otimes \mathbb{Z} \rightarrow 0
$$


where the tensor products are over $\mathbb{Z}$. However, this is obviously isomorphic to another bar complex,

$$
\cdots \rightarrow \mathbb{F}_{\ell} \otimes\left(\mathbb{F}_{\ell} \otimes R\right) \otimes\left(\mathbb{F}_{\ell} \otimes R\right) \otimes \mathbb{F}_{\ell} \rightarrow \mathbb{F}_{\ell} \otimes\left(\mathbb{F}_{\ell} \otimes R\right) \otimes \mathbb{F}_{\ell} \rightarrow \mathbb{F}_{\ell} \otimes \mathbb{F}_{\ell} \rightarrow 0,
$$

which is the bar complex for computing $\operatorname{Tor}_{*}^{\mathbb{F}_{\ell} \otimes R}\left(\mathbb{F}_{\ell}, \mathbb{F}_{\ell}\right)$.

If $\overline{\mathbb{F}}_{\ell}$ is an algebraic closure of $\mathbb{F}_{\ell}$, then $\overline{\mathbb{F}}_{\ell} \otimes-$ is an exact functor from $\mathbb{F}_{\ell} \otimes R-$ modules to $\overline{\mathbb{F}}_{\ell} \otimes R$-modules that takes projective objects to projective objects and commutes with tensor products. Therefore,

$$
\overline{\mathbb{F}}_{\ell} \otimes \operatorname{Tor}_{*}^{\mathbb{F}_{\ell} \otimes R}\left(\mathbb{F}_{\ell}, \mathbb{F}_{\ell}\right) \cong \operatorname{Tor}_{*}^{\overline{\mathbb{F}}_{\ell} \otimes R}\left(\overline{\mathbb{F}}_{\ell}, \overline{\mathbb{F}}_{\ell}\right) .
$$

The characteristic $\ell$ does not divide $|\mathcal{G}|$, so $\overline{\mathbb{F}}_{\ell} \otimes R$ is isomorphic, by basic character theory, to the ring of $\overline{\mathbb{F}}_{\ell}$-valued class functions on $\mathcal{G}$. In other words, $\overline{\mathbb{F}}_{\ell} \otimes R \cong \prod \overline{\mathbb{F}}_{\ell}$. This ring is semisimple, and so it has no higher Tor groups.

Because $\overline{\mathbb{F}}_{\ell} \otimes(-)$ is faithful on $\mathbb{F}_{\ell}$-modules, this shows that

$$
0 \cong \operatorname{Tor}_{*}^{\mathbb{F}_{\ell} \otimes R}\left(\mathbb{F}_{\ell}, \mathbb{F}_{\ell}\right) \cong \operatorname{Tor}_{*}^{R}\left(\mathbb{F}_{\ell}, \mathbb{Z}\right)
$$

for $*>0$, as desired.

Corollary 45 If $\mathcal{G}$ is a nilpotent profinite group, the map $R\left[\mathcal{G}_{\ell}^{\wedge}\right] \rightarrow R[\mathcal{G}]$ is a multiplicative $\mathbb{H} \mathbb{F}_{\ell}$-equivalence.

Proof Because $\mathcal{G}$ is nilpotent and profinite, it is an inverse limit of nilpotent finite groups, each of which is canonically a product of its (unique) Sylow subgroups [16, Exercise 8.8]. This makes $\mathcal{G}$ an inverse limit of groups of the form $K_{1} \times K_{2}$, where $K_{1}$ ranges over the $\ell$-group quotients of $\mathcal{G}$ and $K_{2}$ ranges over the prime-to- $\ell$ quotients. Therefore, $\mathcal{G}$ can be canonically expressed as a direct product $\mathcal{G} \cong \mathcal{G}_{\ell}^{\wedge} \times H$, where $H$ is the inverse limit of groups whose orders are prime to $\ell$. The result now follows from Lemma 43.

\section{Localization in the case of the Heisenberg group}

Let $H$ be the integer Heisenberg group; ie, the group of $3 \times 3$ upper triangular matrices of the form:

$$
\left[\begin{array}{lll}
1 & \mathbb{Z} & \mathbb{Z} \\
0 & 1 & \mathbb{Z} \\
0 & 0 & 1
\end{array}\right]
$$

Alternatively, $H$ has presentation

$$
\langle x, y, z \mid[x, z]=[y, z]=1,[x, y]=z\rangle .
$$




\subsection{The representation ring of the Heisenberg group}

The structure of the set of irreducible representations of the group $H$ was made explicit in [12]. The main result of this section is to compute the product structure in the representation ring. Recall that $\mu_{\infty}$ is the set of roots of unity in $\mathbb{C}$, and $\mu_{p}$ is the set of roots of $p$-power order.

Proposition 46 The space $H^{*}$ of irreducible unitary representations of $H_{a b}$, the abelianization of $H$, is the torus $S^{1} \times S^{1}$. As a space acted on by $H^{*}$ by tensor product,

$$
\operatorname{Irr}(H) \cong \coprod_{\zeta \in \mu_{\infty}}\left(S^{1} \times S^{1} / I_{\zeta}\right)\left[V_{\zeta}\right]
$$

where $I_{\zeta}$ is the subgroup generated by $(\zeta, 1)$ and $(1, \zeta)$. The $\left[V_{\zeta}\right]$ are a specific family of orbit representatives, with $\left[V_{1}\right]$ the trivial representation. The tensor product on $\operatorname{Irr}(H)$ is given as follows.

If $\zeta$ and $\zeta^{\prime}$ have relatively prime orders, $\left[V_{\zeta}\right]\left[V_{\zeta^{\prime}}\right]=\left[V_{\zeta \zeta^{\prime}}\right]$. Otherwise, suppose $\zeta$ has order $p^{k}, \zeta^{\prime}$ has order $p^{l}$, and $\zeta \zeta^{\prime}$ has order $p^{m}$, with $k \geq l$. Then the product is as follows.

$$
\left[V_{\zeta}\right]\left[V_{\zeta^{\prime}}\right] \cong\left\{\begin{array}{cc}
p^{l}\left[V_{\zeta \zeta^{\prime}}\right] & \text { if } k=m \\
p^{m}\left[\sum_{i, j=1}^{p^{k-m}}\left(\zeta^{i}, \zeta^{j}\right)\right]\left[V_{\zeta \zeta^{\prime}}\right] & \text { if } k>m
\end{array}\right.
$$

Proof We will first recall the isomorphism classification of irreducible unitary representations of $H$. In [12, Theorem 4], it is proved that simple representations of $H$ of degree $r$ are classified by triples $(\alpha, \beta, \zeta)$ of nonzero complex numbers, with $\zeta$ a primitive $r$ 'th root of unity, such that $(\alpha, \beta, \zeta) \sim\left(\alpha^{\prime}, \beta^{\prime}, \zeta^{\prime}\right)$ if and only if $\alpha^{r}=\left(\alpha^{\prime}\right)^{r}$, $\zeta=\zeta^{\prime}$, and $(\beta)^{r}=\left(\beta^{\prime}\right)^{r}$. (The triple $(\alpha, \beta, \zeta)$ corresponds to $\left[\alpha^{r}, \beta, \zeta\right]$ in the notation of [12]; the notation we use makes multiplicative relations simpler.)

More specifically, the representation $(\alpha, \beta, \zeta)$, for $\zeta$ a primitive $r$ 'th root of unity, is induced from the closed subgroup $H_{r}$ generated by $\left\langle x^{r}, y, z\right\rangle$. Because $\left[x^{r}, y\right]=z^{r}$ in $H$, we can define a character of $H_{r}$ by

$$
\omega: x^{r} \mapsto \alpha^{r}, y \mapsto \beta, z \mapsto \zeta .
$$

Then $(\alpha, \beta, \zeta)$ corresponds to the representation of $H$ induced from $\omega$. This representation is characterized by the properties that on this representation, $z$ acts by the scalar $\zeta$, the action of $x$ has an eigenvalue of $\alpha$, and the action of $y$ has an eigenvalue of $\beta$. Specifically, the group of characters of $H$ is the collection of representations $(\alpha, \beta, 1)$ for $\alpha, \beta \in \mathbb{C}^{\times} \times \mathbb{C}^{\times}$. In this representation, $x$ acts by $\alpha$ and $y$ acts by $\beta$. This character admits a unitary structure if and only if $(\alpha, \beta) \in S^{1} \times S^{1}$. 
This characterization of irreducible representations allows us to determine the multiplicative structure in the representation ring. If $g$ acts on $V$ with eigenvalues $\left\{\alpha_{i}\right\}_{i}$ and on $W$ with eigenvalues $\left\{\alpha_{j}^{\prime}\right\}_{j}$, then it acts on $V \otimes W$ with eigenvalues $\left\{\alpha_{i} \alpha_{j}^{\prime}\right\}_{i, j}$.

For example, under tensor product we have the formula

$$
(\alpha, \beta, \zeta) \otimes\left(\alpha^{\prime}, \beta^{\prime}, 1\right) \cong\left(\alpha \alpha^{\prime}, \beta \beta^{\prime}, \zeta\right)
$$

because in the tensor product representation the element $z$ still acts by the scalar $\zeta$ and the elements $x$ and $y$ have eigenvectors with eigenvalues $\alpha \alpha^{\prime}$ and $\beta \beta^{\prime}$, respectively.

By construction, if $\alpha$ and $\beta$ lie on the unit circle, the character $\omega$ admits a unitary structure, and hence so does the induced representation $(\alpha, \beta, \zeta)$. Conversely, $\alpha$ and $\beta$ appear as eigenvalues in $(\alpha, \beta, \zeta)$, so this representation admits a unitary structure only if $\alpha$ and $\beta$ lie on the unit circle.

The above considerations give the following decomposition of $\operatorname{Irr}(H)$ as an $H^{*}$-space. Explicity,

$$
\operatorname{Irr}(H) \cong \coprod_{\zeta \in \mu_{\infty}}\left(S^{1} \times S^{1} /\langle\zeta\rangle \times\langle\zeta\rangle\right) \cdot(1,1, \zeta)
$$

The homotopy groups of $R[H]$ are the homology groups of this space. In order to determine the ring structure, it remains to identify the tensor product $(1,1, \zeta) \otimes\left(1,1, \zeta^{\prime}\right)$.

Note that $z$ acts by the scalar $\zeta \zeta^{\prime}$ on $(1,1, \zeta) \otimes\left(1,1, \zeta^{\prime}\right)$, and so this tensor product is a direct sum of representations of the form $\left(\alpha, \beta, \zeta \zeta^{\prime}\right)$. If $\zeta$ and $\zeta^{\prime}$ have relatively prime orders, $(1,1, \zeta) \otimes\left(1,1, \zeta^{\prime}\right)$ has the same dimension as $\left(1,1, \zeta \zeta^{\prime}\right)$ and contains eigenvectors of eigenvalue 1 for both $x$ and $y$, so $\left(1,1, \zeta \zeta^{\prime}\right)=(1,1, \zeta) \otimes\left(1,1, \zeta^{\prime}\right)$.

Let $r$ and $s$ be the orders of $\zeta$ and $\zeta^{\prime}$ respectively. Let $t$ be the order of $\zeta \zeta^{\prime}$, and $\eta$ be a primitive generator for the group $\left\langle\zeta, \zeta^{\prime}\right\rangle$ with order $d t$ such that $\eta^{d}=\zeta \zeta^{\prime}$.

The eigenvalues of $x$ and $y$ in $(1,1, \zeta)$ are $\left\{\zeta^{i}\right\}_{i=1}^{r}$, and similarly for $\left(1,1, \zeta^{\prime}\right)$. Therefore, we have that

$$
(1,1, \zeta) \otimes\left(1,1, \zeta^{\prime}\right)=\sum_{i, j=1}^{d} a_{i, j}\left(\eta^{i}, \eta^{j}, \zeta \zeta^{\prime}\right)
$$

for some coefficients $a_{i, j}$.

The representation $(1,1, \zeta)$ is invariant under tensoring with the characters $(\zeta, 1,1)$ and $(1, \zeta, 1)$, so the same is true for is $(1,1, \zeta) \otimes\left(1,1, \zeta^{\prime}\right)$, and similarly for multiplying by $\left(\zeta^{\prime}, 1,1\right)$ and $\left(1, \zeta^{\prime}, 1\right)$. This forces the coefficients $a_{i, j}$ to be constant in $i$ and $j$. 
As a result, calculating the dimensions of both sides, we find that

$$
(1,1, \zeta) \otimes\left(1,1, \zeta^{\prime}\right)=\frac{r s}{t d^{2}} \sum_{i, j=1}^{d}\left(\eta^{i}, \eta^{j}, \zeta \zeta^{\prime}\right)
$$

The formulation of the proposition can be recovered by writing $\left[V_{\zeta}\right]$ for $(1,1, \zeta)$ and examining the following cases.

If $\zeta$ and $\zeta^{\prime}$ have relatively prime orders, then $t=r s$ and $d=1$, giving the formula

$$
(1,1, \zeta) \otimes\left(1,1, \zeta^{\prime}\right)=\left(1,1, \zeta \zeta^{\prime}\right)
$$

Therefore, it suffices to now determine the multiplication rule when the orders of $\zeta$ and $\zeta^{\prime}$ are prime powers.

Suppose $r=p^{k}, s=p^{l}$, and $t=p^{m}$, with $k \geq l$; this forces $d=p^{k-m}$. If $k=m$, then $t=p^{k}$ and $d=1$, giving the formula

$$
(1,1, \zeta) \otimes\left(1,1, \zeta^{\prime}\right)=p^{l}\left(1,1, \zeta \zeta^{\prime}\right)
$$

Finally, if $k>m$, then we must have $k=l$, giving the fomula

$$
(1,1, \zeta) \otimes\left(1,1, \zeta^{\prime}\right)=p^{m} \sum_{i, j=1}^{p^{k-m}}\left(\eta^{i}, \eta^{j}, \zeta \zeta^{\prime}\right),
$$

as desired.

\subsection{A Bousfield equivalent algebra to $R[H]$}

The results of the previous section allow us to compute the homotopy groups of the $\mathbb{H Z}$-algebra $R[H]$, which are isomorphic by Proposition 38 to the homology groups of $\operatorname{Irr}(H)$.

For convenience, we will write the order of a root of unity $\zeta$ as $|\zeta|$.

Corollary $47 \operatorname{Irr}(H)$ has the following homology groups:

$$
H_{*}(\operatorname{Irr}(H)) \cong \begin{cases}\oplus \zeta \in \mu_{\infty} \mathbb{Z} a_{\zeta} & \text { in dimension 0 } \\ \bigoplus \zeta \in \mu_{\infty}\left[\mathbb{Z} b_{\zeta} \oplus \mathbb{Z} c_{\zeta}\right] & \text { in dimension 1 } \\ \bigoplus \zeta \in \mu_{\infty} \mathbb{Z} d_{\zeta} & \text { in dimension 2 } \\ 0 & \text { otherwise. }\end{cases}
$$


The products are given as follows.

$$
\begin{aligned}
a_{\zeta} a_{\zeta^{\prime}} & =\frac{|\zeta|\left|\zeta^{\prime}\right|}{\left|\zeta \zeta^{\prime}\right|} a_{\zeta \zeta^{\prime}} \\
a_{\zeta} b_{\zeta^{\prime}} & =|\zeta| b_{\zeta \zeta^{\prime}} \\
a_{\zeta} c_{\zeta^{\prime}} & =|\zeta| c_{\zeta \zeta^{\prime}} \\
a_{\zeta} d_{\zeta^{\prime}} & =\frac{|\zeta|\left|\zeta \zeta^{\prime}\right|}{\left|\zeta^{\prime}\right|} d_{\zeta \zeta^{\prime}} \\
b_{\zeta} c_{\zeta^{\prime}} & =\left|\zeta \zeta^{\prime}\right| d_{\zeta \zeta^{\prime}}
\end{aligned}
$$

Proof Write $a_{\zeta}$ for the element in $H_{0}(\operatorname{Irr}(H))$ corresponding to the torus containing $\left[V_{\zeta}\right]$. The multiplication formula for $V_{\zeta}$ and $V_{\zeta}^{\prime}$ implies the formula for $a_{\zeta} a_{\zeta^{\prime}}$.

There are unique choices of generators for the homology of the torus containing $\left[V_{\zeta}\right]$ such that $a_{\zeta} b_{1}=|\zeta| b_{\zeta}, a_{\zeta} c_{1}=|\zeta| c_{\zeta}$, and $b_{1} c_{1}=d_{1}$, due to the structure of $\operatorname{Irr}(H)$ as a set acted on by $H^{*}$.

The remaining product identities can all be derived from these because the homology is torsion-free.

The remainder of this section is devoted to showing that at the prime $\ell$, much of the homotopy of $R=R[H]$ vanishes after Bousfield localization. Specifically, we will exhibit a subobject $\widetilde{R}$ of $R$ such that the Bousfield localization of $R$ is the same as that of $\widetilde{R}$.

Consider the subspace

$$
X=\coprod_{\zeta \in \mu_{\ell} \infty}\left(\frac{S^{1} \times S^{1}}{\langle\zeta\rangle \times\langle\zeta\rangle}\right)\left[V_{\zeta}\right]
$$

of $\operatorname{Irr}(H)$. This subspace consists of representations in which the central element acts by a $\ell$-power root of unity, and is closed under $\otimes$ in the sense that the tensor product of any two representations in $X$ is a direct sum of representations in $X$.

Definition 48 Define $\widetilde{R}$ to be the subobject $\mathcal{S} p\left(\overline{\left(\operatorname{Sym}^{\infty} X\right)}\right)$ of $R[H]$.

The map of abelian topological semirings $\operatorname{Sym}^{\infty}(X) \rightarrow \operatorname{Sym}^{\infty}\left(\operatorname{Irr}(G)_{+}\right) \simeq \operatorname{Rep}(G)$ induces a map of commutative $\mathbb{S}$-algebras $\widetilde{R} \rightarrow R$. On homotopy groups, this map is the map $H_{*}(X) \rightarrow H_{*}(\operatorname{Irr}(H))$.

Both $R_{*}$ and $\widetilde{R}_{*}$ have no torsion. If we define $R_{\ell}=\operatorname{holim} R / \ell^{n}, \pi_{*}\left(R_{\ell}\right) \cong$ $\lim \pi_{*}(R) / \ell^{n}$, and the map $R \rightarrow R_{\ell}$ gives an isomorphism after taking the smash 
product $-\wedge_{\mathbb{H Z}} \mathbb{M} \mathbb{F}_{\ell}$. By Proposition 12 , the map $R \rightarrow R_{\ell}$ is a multiplicative $\mathbb{H F}_{\ell}-$ equivalence. Similarly, we can form $\widetilde{R}_{\ell}$, and the map $\widetilde{R} \rightarrow \widetilde{R}_{\ell}$ is a multiplicative $\mathbb{M} \mathbb{F}_{\ell}$-equivalence.

Proposition 49 There is a map $R \rightarrow \widetilde{R}_{\ell}$ of commutative $\mathbb{S}$-algebras which is a multiplicative $\mathbb{\because} \mathbb{F}_{\ell}$-equivalence.

Proof Suppose $\zeta$ is a root of unity of order relatively prime to $\ell$. Then in $\pi_{0}\left(R_{\ell}\right)$, there is an element $a_{\zeta} /|\zeta|$ such that $\left(a_{\zeta} /|\zeta|\right)^{|\zeta|}=1$. Because $|\zeta|$ is invertible in $\pi_{0}\left(R_{\ell}\right)$, we can form the idempotent

$$
e_{\zeta}=\frac{1}{|\zeta|} \sum_{k=1}^{|\zeta|}\left(\frac{a_{\zeta}}{|\zeta|}\right)^{k}=\frac{1}{|\zeta|} \sum_{k=1}^{|\zeta|} \frac{a_{\zeta^{k}}}{\left|\zeta^{k}\right|} .
$$

This idempotent is an element of the group ring generated by $a_{\zeta} /|\zeta|$ corresponding to the trivial representation. The kernel of multiplication by this idempotent is precisely the ideal generated by $1-a_{\zeta} /|\zeta|$, or equivalently $|\zeta|-a_{\zeta}$.

The element $e_{\zeta} \in \pi_{0}\left(R_{\ell}\right)=\left[R_{\ell}, R_{\ell}\right]_{R_{\ell}}$ can be viewed as a homotopy class of self-maps of $R_{\ell}$. We can then form the homotopy colimit

$$
e_{\zeta} R_{\ell}=\operatorname{hocolim}\left\{R_{\ell} \stackrel{e_{\zeta}}{\rightarrow} R_{\ell} \stackrel{e_{\zeta}}{\rightarrow} R_{\ell} \stackrel{e_{\zeta}}{\rightarrow} \cdots\right\}
$$

whose homotopy groups are $e_{\zeta} \pi_{*}\left(R_{\ell}\right)$.

By the Künneth spectral sequence of [7, Theorem IV.4.1], we have

$$
\pi_{*}\left(M \underset{R_{\ell}}{\wedge} e_{\zeta} R_{\ell}\right) \cong e_{\zeta} \pi_{*} M
$$

for any $R_{\ell}$-module $M$. In particular, the map

$$
e_{\zeta} R_{\ell} \underset{R_{\ell}}{\wedge} R_{\ell} \rightarrow e_{\zeta} R_{\ell} \underset{R_{\ell}}{\wedge} e_{\zeta} R_{\ell}
$$

is an equivalence. Therefore, the map $R_{\ell} \rightarrow e_{\zeta} R_{\ell}$ is an $\left(e_{\zeta} R_{\ell}\right)$-equivalence of $R_{\ell-}$ modules. Additionally, $e_{\zeta} R_{\ell}$ is $\left(e_{\zeta} R_{\ell}\right)$-local, since any map $f: M \rightarrow e_{\zeta} R_{\ell}$ factors as

$$
M \stackrel{\eta \wedge 1}{\rightarrow} e_{\zeta} R_{\ell} \underset{R_{\ell}}{\wedge} \stackrel{1 \wedge f}{\rightarrow} e_{\zeta} R_{\ell} \underset{R_{\ell}}{\wedge} e_{\zeta} R_{\ell} \stackrel{\mu}{\rightarrow} e_{\zeta} R_{\ell} .
$$

Here $\eta$ is the unit and $\mu$ is the multiplication of the $R_{\ell}$-ring spectrum $e_{\zeta} R_{\ell}$. Therefore, $e_{\zeta} R_{\ell}$ is a Bousfield localization of $R_{\ell}$, and admits the structure of a commutative $R_{\ell}$-algebra by [7, Theorem VIII.2.2]. 
We also have

$$
\pi_{*}\left(\mathbb{H} \mathbb{F}_{\ell} \underset{R_{\ell}}{\wedge} e_{\zeta} R_{\ell}\right) \cong \pi_{*} \mathbb{H} \mathbb{F}_{\ell},
$$

because the idempotent $e_{\zeta}$ acts by 1 on $\pi_{*} \mathbb{H}\left(\mathbb{F}_{\ell}\right)$. Therefore, there is an isomorphism in the derived category of $R_{\ell}$-modules

$$
\mathbb{H F}{\underset{R}{R_{\ell}}}_{e_{\zeta}} R_{\ell} \simeq \mathbb{H} \mathbb{F}_{\ell}
$$

Taking homotopy colimits in $\zeta$ gives

$$
\left.\mathbb{H} \mathbb{F}_{\ell}{\underset{R_{\ell}}{\wedge}}_{\zeta}^{\operatorname{hocolim} e_{\zeta}} R_{\ell}\right) \simeq \mathbb{M} \mathbb{F}_{\ell}
$$

In other words, applying Proposition 12 with base ring $R_{\ell}$, the map

$$
R_{\ell} \rightarrow \operatorname{hocolim} e_{\zeta} R_{\ell}
$$

induces an equivalence of $\mathbb{H} \mathbb{F}_{\ell}$-localizations.

The map $\pi_{*}\left(R_{\ell}\right) \rightarrow$ colim $e_{\zeta} \pi_{*}\left(R_{\ell}\right)$ is the map that takes the quotient by the ideal generated by all elements $a_{\zeta}-|\zeta|$, where $\zeta$ varies over roots of unity relatively prime to $\ell$. The composite map $\pi_{*}\left(\widetilde{R}_{\ell}\right) \rightarrow \pi_{*}\left(R_{\ell}\right) \rightarrow$ hocolim $e_{\zeta} \pi_{*}\left(R_{\ell}\right)$ is an isomorphism. The map $\widetilde{R}_{\ell} \rightarrow$ hocolim $e_{\zeta} R_{\ell}$ therefore is an equivalence.

Choosing a homotopy inverse, we can then form the composite map

$$
R \rightarrow R_{\ell} \rightarrow \operatorname{hocolim} e_{\zeta} R_{\ell} \stackrel{\sim}{\longrightarrow} \widetilde{R}_{\ell} .
$$

This is a composite of multiplicative $\mathbb{A} \mathbb{F}_{\ell}$-equivalences, and hence is a multiplicative $\mathbb{M} \mathbb{F}_{\ell}$-equivalence by Lemma 13.

\subsection{Localized homotopy of $R[H]$}

In the previous section, we found an object $\widetilde{R}$ whose $\mathbb{U F}_{\ell}$-localization is equivalent to the localization of $R[H]$. We will now show that the object $\widetilde{R}_{\ell}=\operatorname{holim} \widetilde{R} / \ell^{n}$ is already $\mathbb{H} \mathbb{F}_{\ell}$-local.

Theorem $50 \quad \widetilde{R}_{\ell}$ is $\mathbb{H} \mathbb{F}_{\ell}$-local as an $\widetilde{R}$-module.

Corollary 51 The homology of the $\mathbb{H} \mathbb{F}_{\ell}$-localization of $R[H]$ is the same as the homotopy of $\widetilde{R}_{\ell}$ :

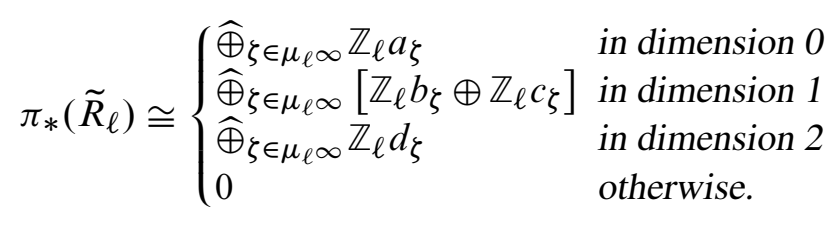


The products are given as in Corollary 47.

The notation $\widehat{\oplus}$ denotes direct sum in the category of $\ell$-complete modules:

$$
\widehat{\oplus} M_{\alpha}=\lim \oplus M_{\alpha} / \ell^{n} \text {. }
$$

Proof Recall from Section 2 that $\mathbb{H}_{\ell}$-local objects are closed under homotopy limits, $\mathbb{M} \mathbb{F}_{\ell}$-modules, and exact triangles. We have exact triangles

$$
\tilde{R}_{\ell} / \ell \stackrel{\ell^{n}}{\rightarrow} \tilde{R}_{\ell} / \ell^{n+1} \longrightarrow \tilde{R}_{\ell} / \ell^{n}
$$

for all $n$. We will demonstrate that $\widetilde{R}_{\ell} / \ell$ is $\mathbb{H} \mathbb{F}_{\ell}$-nilpotent. It then follows that $\widetilde{R}_{\ell} / \ell^{n}$ is $\mathbb{H} \mathbb{F}_{\ell}$-nilpotent for all $n$. Because $\widetilde{R}_{\ell} \simeq$ holim $\widetilde{R}_{\ell} / \ell^{n}, \widetilde{R}_{\ell}$ is $\mathbb{M} \mathbb{F}_{\ell}$-local.

Consider the kernel $I$ of the map $\pi_{0}\left(\widetilde{R}_{\ell}\right) \rightarrow \mathbb{F}_{\ell}$. I is generated by $\ell$ and by the elements $a_{\zeta}$ for $\zeta \in \mu_{\ell}, \zeta \neq 1$. In particular, the multiplication rules show that $\ell$ divides $a_{\zeta} a_{\zeta^{\prime}}$ for any $\zeta, \zeta^{\prime} \neq 1$, so $I^{2} \subset(\ell)$.

If $M$ is any module over $\pi_{0}\left(\widetilde{R}_{\ell}\right)$ that is acted on trivially by $\ell$, there is a short exact sequence of $\pi_{0}\left(\widetilde{R}_{\ell} / \ell\right)$-modules

$$
0 \rightarrow I M \rightarrow M \rightarrow M / I M \rightarrow 0 .
$$

Both $I M$ and $M / I M$ are acted on trivially by $I$. They are modules over $\pi_{0}\left(\widetilde{R}_{\ell}\right) / I \cong$ $\mathbb{F}_{\ell}$, and therefore the Eilenberg-MacLane module spectra $\mathbb{H}(I M)$ and $\mathbb{H}(M / I M)$ are local as $\widetilde{R}_{\ell}$-modules. Because local modules are closed under exact triangles, $\mathbb{H} M$ is $\mathbb{H F} \mathbb{F}_{\ell}$-local as an $\widetilde{R}_{\ell}$-module.

Consider the Postnikov tower for $\widetilde{R}_{\ell} / \ell$, which terminates after degree 2.

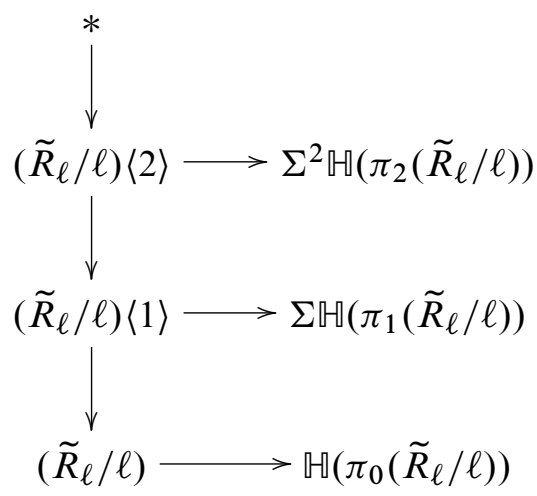

The Eilenberg-MacLane objects are modules over $\mathbb{H} \pi_{0}\left(\tilde{R}_{\ell}\right)$ and are acted on trivially by $\ell$. They are therefore $\mathbb{M} \mathbb{F}_{\ell}$-local as $\widetilde{R}_{\ell}$-modules. Consequently, $\widetilde{R}_{\ell} / \ell$ is $\mathbb{H} \mathbb{F}_{\ell-}$ local, as desired. 


\subsection{Derived completion of $R[H]$}

In this section, we show that the computation of the homotopy groups of the $\mathbb{H F}_{\ell}-$ localization of $R\left[H_{\ell}^{\wedge}\right]$ from the previous section is actually a computation of the homotopy of the derived completion of $R\left[H_{\ell}^{\wedge}\right]$, as explained in the introduction. Consider the $\mathbb{A} \mathbb{F}_{\ell}$-nilpotent completion of the $\mathbb{S}$-algebra $R\left[H_{\ell}^{\wedge}\right]$. By Lemma 18 it is equivalent to the $\mathbb{H F}_{\ell}$-nilpotent completion of the $\mathbb{S}$-algebra $\widetilde{R}$ constructed in previous sections. Our claim is as follows:

Theorem 52 The pro-object $\left\{\widetilde{R} / \ell^{n}\right\}$ is an $\mathbb{H} \mathbb{F}_{\ell}$-nilpotent completion of $\widetilde{R}$.

Proof The objects $\widetilde{R} / \ell^{n}$ are $\mathbb{H F}_{\ell}$-nilpotent, as shown in Corollary 51.

Let $X$ be an $\mathbb{M} \mathbb{F}_{\ell}$-module. For any $n$, consider the following map of cofiber sequences.

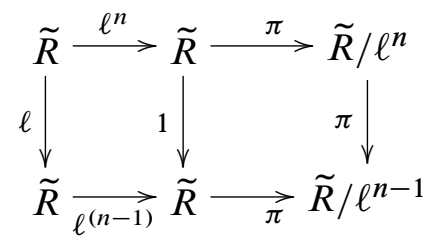

Apply $[-, X]_{\widetilde{R}}$ to the above diagram. Multiplication by $\ell$ is zero on $\pi_{*} X$, so we get the following map of short exact sequences.

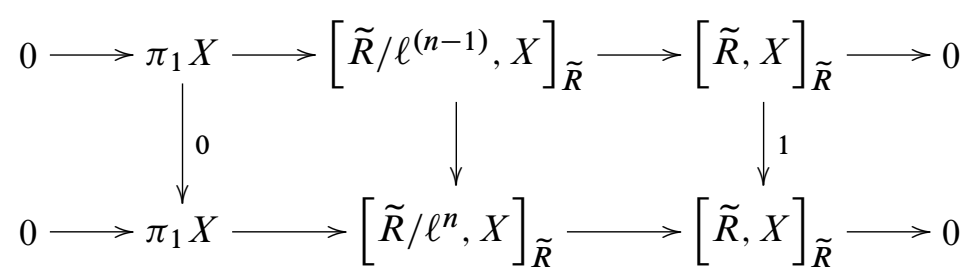

Taking colimits in $n$ gives the desired result.

As a result, the homotopy groups of $\widetilde{R}_{\mathfrak{W} \mathbb{F}_{\ell}}^{\wedge}$ are the pro-groups $\left\{\pi_{*}\left(\widetilde{R} / \ell^{n}\right)\right\}$. The ring $\widetilde{R}$ has no $\ell$-torsion, so $\pi_{*}\left(\widetilde{R} / \ell^{n}\right) \cong \pi_{*}(\widetilde{R}) / \ell^{n}$, which are the same as the homotopy groups of $\pi_{*}\left(\widetilde{R}_{\ell}\right)$ viewed as a pro-system.

\section{References}

[1] J F Adams, Stable homotopy and generalised homology, University of Chicago Press, Chicago, Ill. (1974) MR0402720

[2] A Baker, A Lazarev, On the Adams spectral sequence for $R$-modules, Algebr. Geom. Topol. 1 (2001) 173-199 MR1823498 
[3] A K Bousfield, The localization of spectra with respect to homology, Topology 18 (1979) 257-281 MR551009

[4] C Bray, On the cohomology of some representation rings, $\mathrm{PhD}$ thesis, Stanford University (1999)

[5] G Carlsson, An Adams-type spectral sequence for change of rings, Houston J. Math. 4 (1978) 541-550 MR523612

[6] G Carlsson, Structured stable homotopy theory and the descent problem for the algebraic $K$-theory of fields, preprint (2003)

[7] A D Elmendorf, I Kriz, M A Mandell, J P May, Rings, modules, and algebras in stable homotopy theory, Mathematical Surveys and Monographs 47, American Mathematical Society, Providence, RI (1997) MR1417719

[8] M Hovey, J H Palmieri, N P Strickland, Axiomatic stable homotopy theory, Mem. Amer. Math. Soc. 128 (1997) x+114 MR1388895

[9] A Lubotzky, A R Magid, Varieties of representations of finitely generated groups, Mem. Amer. Math. Soc. 58 (1985) xi+117 MR818915

[10] M Lydakis, Smash products and $\Gamma$-spaces, Math. Proc. Cambridge Philos. Soc. 126 (1999) 311-328 MR1670245

[11] M A Mandell, J P May, S Schwede, B Shipley, Model categories of diagram spectra, Proc. London Math. Soc. (3) 82 (2001) 441-512 MR1806878

[12] C Nunley, A Magid, Simple representations of the integral Heisenberg group, from: "Classical groups and related topics (Beijing, 1987)", Contemp. Math. 82, Amer. Math. Soc., Providence, RI (1989) 89-96 MR982280

[13] S Schwede, Stable homotopical algebra and $\Gamma$-spaces, Math. Proc. Cambridge Philos. Soc. 126 (1999) 329-356 MR1670249

[14] S Schwede, S-modules and symmetric spectra, Math. Ann. 319 (2001) 517-532 MR1819881

[15] G Segal, Categories and cohomology theories, Topology 13 (1974) 293-312 MR0353298

[16] J-P Serre, Linear representations of finite groups, Springer, New York (1977) MR0450380

Department of Mathematics, Massachusetts Institute of Technology

Cambridge MA 02139, USA

tlawson@math.mit.edu

Received: 11 April 2005 Revised: 31 October 2005

Algebraic $\& \mathcal{G}$ Geometric Topology, Volume 6 (2006) 